\title{
On Hardy and BMO Spaces for Grushin Operator
}

\author{
Jacek Dziubański ${ }^{1}$ • K. Jotsaroop ${ }^{2}$
}

Received: 13 May 2015 / Published online: 9 December 2015

(C) The Author(s) 2015. This article is published with open access at Springerlink.com

\begin{abstract}
We study Hardy and BMO spaces associated with the Grushin operator. We first prove atomic and maximal functions characterizations of the Hardy space. Further we establish a version of Fefferman-Stein decomposition of BMO functions associated with the Grushin operator and then obtain a Riesz transforms characterization of the Hardy space.
\end{abstract}

Keywords Grushin operator · Heat kernel · Hardy space - Maximal operator . Atomic decomposition · Riesz transforms · BMO space

Mathematics Subject Classification Primary 42B30 - Secondary 43A85 - 42B25 . $42 \mathrm{~B} 20 \cdot 35 \mathrm{~J} 70 \cdot 22 \mathrm{E} 25$

Communicated by Hans G. Feichtinger.

$凶 \quad$ Jacek Dziubański

Jacek.Dziubanski@math.uni.wroc.pl

K. Jotsaroop

jotsaroop.kaur@unimib.it

1 Instytut Matematyczny, Uniwersytet Wrocławski, Pl. Grunwaldzki 2/4, 50-384 Wrocław, Poland

2 Department of Mathematics and its Applications, University of Milan-Bicocca, Milan, Italy 


\section{Introduction and Statement of the Results}

\subsection{Grushin Operator}

On $\mathbb{R}^{n+1}=\mathbb{R}^{n} \times \mathbb{R}$ we consider the Grushin operator

$$
\mathcal{L}=-\sum_{j=1}^{n}\left(\frac{\partial^{2}}{\partial x_{j}^{\prime 2}}+\left(x_{j}^{\prime}\right)^{2} \frac{\partial^{2}}{\partial x^{\prime \prime 2}}\right)=-\Delta_{x^{\prime}}-\left|x^{\prime}\right|^{2} \frac{\partial^{2}}{\partial x^{\prime \prime 2}},
$$

where $\mathbb{R}^{n} \times \mathbb{R} \ni \mathbf{x}=\left(x^{\prime}, x^{\prime \prime}\right), x^{\prime}=\left(x_{1}^{\prime}, x_{2}^{\prime}, \ldots, x_{n}^{\prime}\right) \in \mathbb{R}^{n}, x^{\prime \prime} \in \mathbb{R}$. The operator $\mathcal{L}$ is homogeneous of degree 2 with respect to the dilations

$$
\delta_{t} \mathbf{x}=\left(t x^{\prime}, t^{2} x^{\prime \prime}\right),
$$

that is, $\mathcal{L}\left(f \circ \delta_{t}\right)(\mathbf{x})=t^{2}(\mathcal{L} \mathcal{F})\left(\delta_{t} \mathbf{x}\right)$. It is well known $\mathcal{L}$ is a hypoelliptic operator. It is related to the Heisenberg group $\mathbb{H}_{n}$. Actually the Grushin operator $\mathcal{L}$ is the image of a sub-Laplacian $L$ associated to $\mathbb{H}_{n}$ under a representation $\pi$ acting on functions on $\mathbb{R}^{n+1}$. In fact we make use of this relation to prove some crucial estimates on some kernels related to $\mathcal{L}$.

The control distance on $\mathbb{R}^{n+1}$ associated with $\mathcal{L}$ is defined by

$$
d(\mathbf{x}, \mathbf{y})=\sup _{\psi \in \mathcal{D}}|\psi(\mathbf{x})-\psi(\mathbf{y})|
$$

where $\mathcal{D}=\left\{\psi \in W^{1, \infty}\left(\mathbb{R}^{n+1}\right): \sum_{j=1}^{n}\left(\left|\partial_{x_{j}^{\prime}} \psi\right|^{2}+\left|x_{j}^{\prime} \partial_{x^{\prime \prime}} \psi\right|^{2}\right) \leq 1\right\}$. It is homogeneous, that is,

$$
d\left(\delta_{s} \mathbf{x}, \delta_{s} \mathbf{y}\right)=s d(\mathbf{x}, \mathbf{y})
$$

and behaves like:

$$
d(\mathbf{x}, \mathbf{y}) \sim\left|x^{\prime}-y^{\prime}\right|+ \begin{cases}\frac{\left|x^{\prime \prime}-y^{\prime \prime}\right|}{\left|x^{\prime}\right|+\left|y^{\prime}\right|} & \text { if }\left|x^{\prime \prime}-y^{\prime \prime}\right|^{1 / 2} \leq\left|x^{\prime}\right|+\left|y^{\prime}\right|, \\ \left|x^{\prime \prime}-y^{\prime \prime}\right|^{1 / 2} & \text { if }\left|x^{\prime \prime}-y^{\prime \prime}\right|^{1 / 2}>\left|x^{\prime}\right|+\left|y^{\prime}\right|,\end{cases}
$$

see, e.g., [18,20] for details. Clearly,

$$
|f(\mathbf{x})-f(\mathbf{y})| \leq d(\mathbf{x}, \mathbf{y}) \sum_{j=1}^{n}\left(\left\|\partial_{x_{j}^{\prime}} f\right\|_{\infty}+\left\|x_{j}^{\prime} \partial_{x^{\prime \prime}} f\right\|_{\infty}\right)
$$

Let $B(\mathbf{x}, r)=\{\mathbf{y} \in \mathbf{X}: d(\mathbf{x}, \mathbf{y})<r\}$ denote the ball with center $\mathbf{x}$ and radius $r>0$ in the metric $d(\mathbf{x}, \mathbf{y})$ and $|B(\mathbf{x}, r)|$ be its Lebesgue measure volume. Then

$$
|B(\mathbf{x}, r)| \sim r^{n+1} \max \left\{r,\left|x^{\prime}\right|\right\} \sim r^{n+1}\left(r+\left|x^{\prime}\right|\right)
$$

and, consequently, 


$$
\left(\frac{R}{r}\right)^{n+1} \lesssim \frac{|B(\mathbf{x}, R)|}{|B(\mathbf{x}, r)|} \lesssim\left(\frac{R}{r}\right)^{n+2}, \quad R \geq r>0 .
$$

The homogeneity of the distance $d$ implies

$$
\left|B\left(\delta_{s} \mathbf{x}, s r\right)\right|=s^{n+2}|B(\mathbf{x}, r)| .
$$

The space $\mathbf{X}=\mathbb{R}^{n} \times \mathbb{R}$ equipped with the Lebesgue measure $d \mathbf{x}$ and the distance $d(\mathbf{x}, \mathbf{y})$ is the space of homogeneous type in the sense of Coifman-Weiss [7]. It is well known (see e.g., [20]) that $-\mathcal{L}$ generates a semigroup of self-adjoint linear operators $e^{-t \mathcal{L}}$ on $L^{2}(\mathbf{X})$ which has the form

$$
e^{-t \mathcal{L}} f(\mathbf{x})=\int_{\mathbf{X}} H_{t}(\mathbf{x}, \mathbf{y}) f(\mathbf{y}) d \mathbf{y},
$$

where the heat kernel $H_{t}(\mathbf{x}, \mathbf{y})$ satisfies the Gaussian upper bound estimates (see (2.13)).

\subsection{Hardy Space $\boldsymbol{H}_{\mathcal{L}}^{1}$}

Let $\mathcal{M}_{\mathcal{L}} f(x)=\sup _{t>0}\left|e^{-t \mathcal{L}} f(x)\right|$ be the maximal function associated with the semigroup $e^{-t \mathcal{L}}$. The upper Gaussian estimates (2.13) imply that $\mathcal{M}_{\mathcal{L}}$ is bounded on $L^{p}(\mathbf{X})$ for $1<p \leq \infty$ and of weak-type $(1,1)$. We define the Hardy space

$$
\begin{gathered}
H_{\mathcal{L}}^{1}=\left\{f \in L^{1}(\mathbf{X}): \mathcal{M}_{\mathcal{L}} f \in L^{1}(\mathbf{X})\right\} \\
\|f\|_{H_{\mathcal{L}}^{1}}=\left\|\mathcal{M}_{\mathcal{L}} f\right\|_{L^{1}(\mathbf{X})} .
\end{gathered}
$$

Now we define atoms associated to the homogeneous space $\mathbf{X}$.

\subsection{Atoms}

Fix $1<q \leq \infty$. A function $a$ is called a $(1, q)$-atom for the Hardy space $H^{1}(\mathbf{X})$ if there is a ball $B=B(\mathbf{x}, r)=\{\mathbf{y}: d(\mathbf{x}, \mathbf{y})<r\}$ such that

supp $a \subset B$,

$\|a\|_{L^{q}} \leq|B|^{\frac{1}{q}-1}\left(\|a\|_{L^{\infty}} \leq|B|^{-1}\right.$ if $\left.q=\infty\right)$,

$\int a(\mathbf{y}) d \mathbf{y}=0$.

The atomic norm is given by

$$
\|f\|_{H_{\mathrm{atom}, q}^{1}(\mathbf{X}, d)}=\sum\left|\lambda_{j}\right|
$$

where the infimum is taken over all decompositions $f=\sum \lambda_{j} a_{j}, \lambda_{j} \in \mathbb{C}, a_{j}$ are $(1, q)$-atoms for $H^{1}(\mathbf{X})$. 
We are now in a position to state our first result.

Theorem 1.1 For every $q \in(1, \infty]$ the space $H_{\mathcal{L}}^{1}$ admits atomic decomposition and the norms $\|f\|_{H_{\mathcal{L}}^{1}}$ and $\|f\|_{H_{\text {atom, } q}^{1}(\mathbf{X})}$ are equivalent.

\subsection{Riesz Transforms}

The system of Riesz transforms $\mathcal{R}_{j}, j=1,2, \ldots, 2 n$, associated with $\mathcal{L}$ is defined by

$$
\mathcal{R}_{j}=\partial_{x_{j}^{\prime}} \mathcal{L}^{-1 / 2}, \quad \mathcal{R}_{n+j}=x_{j}^{\prime} \partial_{x^{\prime \prime}} \mathcal{L}^{-1 / 2}, \quad j=1,2, \ldots, n
$$

This formal definition has a precise meaning and the operators $\mathcal{R}_{j}$ are CalderónZygmund operators on $\mathbf{X}$. Moreover, $\mathcal{R}_{j}$ are well-defined in the sense of distributions on $L^{1}(\mathbf{X})$ (see Sect. 4). Our second main result is the following theorem.

Theorem 1.2 An $L^{1}(\mathbf{X})$ function $F$ belongs to $H_{\mathcal{L}}^{1}$ if and only if $\mathcal{R}_{j} F \in L^{1}(\mathbf{X})$ for $j=1,2, \ldots, 2 n$. Moreover, there is a constant $C>0$ such that

$$
C^{-1}\|F\|_{H_{\mathcal{L}}^{1}} \leq\|F\|_{L^{1}(\mathbf{X})}+\sum_{j=1}^{2 n}\left\|\mathcal{R}_{j} F\right\|_{L^{1}(\mathbf{X})} \leq C\|F\|_{H_{\mathcal{L}}^{1}} .
$$

The theory of the classical real Hardy spaces on $\mathbb{R}^{n}$ has its origin in studying holomorphic function of one variable. The reader is referred to the very original works: Stein and Weiss [23], Burkholder et al. [2], Fefferman and Stein [11], and Coifman [6]. The spaces are natural extensions of $L^{p}$ spaces and many operators occurring in harmonic analysis, like convolution singular integral operators, are bounded on them. The theory was then extended to the spaces of homogeneous type (see $[8,17,25]$ ). More information about the classical real $H^{p}$ spaces with their characterizations and historical remarks can be also found in [22]. In [13] the authors provide a very general approach to the theory of $H^{1}$ spaces for semigroups of linear operators satisfying Davies-Gaffney estimates and in particular Gaussian bounds. Let us point out, that in the context of semigroups, the classical Hardy spaces can be thought as those associated with the Laplace operator on $\mathbb{R}^{n}$.

In the monograph [12] Folland and Stein study $H^{p}$ spaces on homogeneous nilpotent Lie groups proving equivalence of their definitions by means of maximal functions, square functions, and atoms. Important contributions to the theory of Hardy spaces on homogeneous groups are their characterizations by Riesz transforms proved in Christ and Geller [5]. To this end the authors of [5] extended Uchiyama's theorem (see [26]) about Fefferman-Stein decomposition of BMO functions on $\mathbb{R}^{n}$ to homogeneous nilpotent Lie groups. Let us emphasise that our proof of the Riesz transforms characterization of Hardy space $H_{\mathcal{L}}^{1}$ associated with the Grushin operator (see Theorem 1.2) takes an inspiration from [5]. We make use of the relation between $\mathcal{L}$ and $L$ via the already mentioned representation $\pi$ and transfer the methods of [5] into the space of homogeneous type $\mathbf{X}$. 
Let us also remark that our proof of Theorem 1.1 is based on Uchiyama results [25] about characterization of Hardy spaces on spaces of homogeneous type by maximal functions and atomic decompositions.

The Grushin operator $\mathcal{L}$ we consider here is a special example of operators of the form $\mathbf{L}=-\Delta_{x^{\prime}}-\left|x^{\prime}\right|^{2} \Delta_{x^{\prime \prime}},\left(x^{\prime}, x^{\prime \prime}\right) \in \mathbb{R}^{n} \times \mathbb{R}^{m}$. It seems likely the methods we present here combined with [5] and relation of $\mathbf{L}$ with the Heisenberg-Reiter groups (see, e.g., [18]) will allow to develop the theory of Hardy spaces for $\mathbf{L}$.

\subsection{Organization of the Paper}

In Sect. 2 we describe relation of the Grushin operator and the sub-Laplacian on the Heisenberg group via a unitary representation and derive estimates on the heat kernel of $\exp (-t \mathcal{L})$. Section 3 is devoted to proving Theorem 1.1. In Sect. 4 we study properties of kernels which are obtained as images by the representation of some singular integral kernels on $\mathbb{H}_{n}$. The crucial theorem about decompositions of compactly supported BMO functions by means of singular integrals is stated in Sect. 5 and its proof is completed in Appendixes 1 and 2. The proof of the Riesz transforms characterization of the Hardy space $H_{\mathcal{L}}^{1}$ is presented in Sect. 6.

\section{Relation with the Heisenberg Group}

In this section we describe relation between the Grushin operator $\mathcal{L}$ and the subLaplacian $L$ on the Heisenberg group $\mathbb{H}_{n}$. As we will see $\mathcal{L}$ occurs as an image of $L$ in a special unitary representation $\pi$ of $\mathbb{H}_{n}$ (see $[15,19]$ ). We start this section by recalling basic facts from the analysis on the Heisenberg group.

\subsection{Heisenberg Group}

The Heisenberg group $\mathbb{H}_{n}$ is a Lie group with the underlying manifold $\mathbb{R}^{2 n+1}=$ $\mathbb{R}^{n} \times \mathbb{R}^{n} \times \mathbb{R}$ and the group multiplication

$$
(x, y, t)(u, v, s)=\left(x+u, y+v, t+s+\frac{1}{2}(y \cdot u-x \cdot v)\right),
$$

where $x \cdot y$ is the standard inner product in $\mathbb{R}^{n}$. We shall also denote the elements of the Heisneberg group by $\boldsymbol{x}=(x, y, t)$. Then $\boldsymbol{x}^{-1}=-\boldsymbol{x}=(-x,-y,-t)$. The Lebesgue measure $d \boldsymbol{x}$ on $\mathbb{R}^{2 n+1}$ turns out to be the bi-invariant Haar measure on $\mathbb{H}_{n}$. Clearly, $\mathbb{H}_{n}$ is a homogeneous nilpotent Lie group with dilations $\delta_{s}(x, y, t)=\left(s x, s y, s^{2} t\right)$. We fix a homogeneous norm on $\mathbb{H}_{n}$ to be so called Koranyi norm given by

$$
|\boldsymbol{x}|=|(x, y, t)|=\left(\left(|x|^{2}+|y|^{2}\right)^{2}+16 t^{2}\right)^{1 / 4} .
$$

The function $\mathbb{H}_{n} \ni \boldsymbol{x} \mapsto|\boldsymbol{x}| \in \mathbb{R}_{+} \cup\{0\}$ is smooth away from the origin, homogeneous of degree one, that is, $\left|\delta_{s} \boldsymbol{x}\right|=s|\boldsymbol{x}|$, and symmetric $(|\boldsymbol{x}|=|-\boldsymbol{x}|)$. Moreover, $|\boldsymbol{x} \boldsymbol{y}| \leq$ 
$|\boldsymbol{x}|+|\boldsymbol{y}|$. Clearly, $|(x, y, t)| \sim|x|+|y|+|t|^{1 / 2}$. The homogeneous dimension of $\mathbb{H}_{n}$ is denoted by $D$ and in our case $D=2 n+2$.

We choose the standard basis of the left-invariant vector fields:

$$
X_{j}=\partial_{x_{j}}+\frac{1}{2} y_{j} \partial_{t}, \quad X_{n+j}=\partial_{y_{j}}-\frac{1}{2} x_{j} \partial_{t}, \quad j=1,2, \ldots, n, \quad X_{2 n+1}=\partial_{t},
$$

and the corresponding right-invariant vector fields:

$$
Y_{j}=\partial_{x_{j}}-\frac{1}{2} y_{j} \partial_{t}, \quad Y_{n+j}=\partial_{y_{j}}+\frac{1}{2} x_{j} \partial_{t}, \quad j=1,2, \ldots, n, \quad Y_{2 n+1}=\partial_{t} .
$$

Obviously, for $j=1,2, \ldots, n$ we have

$$
X_{j}=Y_{j}+w_{j} Y_{2 n+1}, \quad X_{n+j}=Y_{n+j}+w_{n+j} Y_{2 n+1}, \quad X_{2 n+1}=Y_{2 n+1},
$$

where $w_{j}(\boldsymbol{x})=w_{j}(x, y, t)=y_{j}, w_{n+j}(\boldsymbol{x})=w_{n+j}(x, y, t)=-x_{j}$.

We apply the usual notation for higher order derivatives (see [12]). If $I=$ $\left(i_{1}, i_{2}, \ldots, i_{2 n+1}\right) \in(\mathbb{N} \cup\{0\})^{2 n+1}$ is a multi-index, we set

$$
X^{I}=X_{1}^{i_{1}} X_{2}^{i_{2}} \ldots X_{2 n+1}^{i_{2 n+1}}, \quad Y^{I}=Y_{1}^{i_{1}} Y_{2}^{i_{2}} \ldots Y_{2 n+1}^{i_{2 n+1}},
$$

$$
d(I)=i_{1}+i_{2}+\cdots+i_{2 n}+2 i_{2 n+1} \text { is the homogeneous degree of } I .
$$

Let $L=-\sum_{k=1}^{2 n} X_{k}^{2}$ denote the left-invariant sub-Laplacian on $\mathbb{H}_{n}$. It is wellknown (see e.g., [27]) that the corresponding heat semigroup $e^{-s L}$ is given by the convolution $e^{-s L} f(x, y, t)=f * h_{s}(x, y, t)$ with a heat kernel $h_{s}(x, y, t)=$ $h_{s}(-x,-y,-t)$ which satisfies

$$
\begin{gathered}
h_{s}(x, y, t)=s^{-D / 2} h_{1}\left(\delta_{s^{-1 / 2}}(x, y, t)\right), \\
s^{-D / 2} e^{-C|(x, y, t)|^{2} / s} \lesssim h_{s}(x, y, t) \lesssim s^{-D / 2} e^{-c|(x, y, t)|^{2} / s} \\
\left|X^{I} Y^{J} h_{s}(x, y, t)\right| \leq C_{I, J} s^{-(D+d(I)+d(J)) / 2} e^{-c|(x, y, t)|^{2} / s} .
\end{gathered}
$$

\subsection{Unitary Representation}

We define the unitary representation of $\mathbb{H}_{n}$ on $L^{2}(\mathbf{X})$ by

$$
\pi_{(x, y, t)} f(\mathbf{x})=\pi_{(x, y, t)} f\left(x^{\prime}, x^{\prime \prime}\right)=f\left(x^{\prime}+y, x^{\prime \prime}+t+\frac{1}{2} x \cdot y+x \cdot x^{\prime}\right)
$$


(cf. Meyer [19]). It is easy to see that

$$
\begin{gathered}
\pi\left(X_{j}\right) f\left(x^{\prime}, x^{\prime \prime}\right)=\pi\left(Y_{j}\right) f\left(x^{\prime}, x^{\prime \prime}\right)=x_{j}^{\prime} \partial_{x^{\prime \prime}} f\left(x^{\prime}, x^{\prime \prime}\right), \quad j=1,2, \ldots, n, \\
\pi\left(X_{n+j}\right) f\left(x^{\prime}, x^{\prime \prime}\right)=\pi\left(Y_{n+j}\right) f\left(x^{\prime}, x^{\prime \prime}\right)=\partial_{x_{j}^{\prime}} f\left(x^{\prime}, x^{\prime \prime}\right), j=1,2, \ldots, n, \\
\pi\left(X_{2 n+1}\right) f\left(x^{\prime}, x^{\prime \prime}\right)=\pi\left(Y_{2 n+1}\right) f\left(x^{\prime}, x^{\prime \prime}\right)=\partial_{x^{\prime \prime}} f\left(x^{\prime}, x^{\prime \prime}\right) .
\end{gathered}
$$

Hence, $\pi(L)=\mathcal{L}$.

For a function $F \in L^{1}\left(\mathbb{H}_{n}\right)$ we set

$$
\pi(F) f\left(x^{\prime}, x^{\prime \prime}\right)=\int_{\mathbb{H}_{n}} F(x, y, t) \pi_{(x, y, t)} f\left(x^{\prime}, x^{\prime \prime}\right)=\int_{\mathbf{X}} \pi(F)(\mathbf{x}, \mathbf{y}) f(\mathbf{y}) d \mathbf{y}
$$

where

$$
\pi(F)(\mathbf{x}, \mathbf{y})=\int_{\mathbb{R}^{n}} F\left(z, y^{\prime}-x^{\prime}, y^{\prime \prime}-x^{\prime \prime}-\frac{1}{2} z \cdot\left(y^{\prime}+x^{\prime}\right)\right) d z
$$

Clearly, if $F \in L^{1}\left(\mathbb{H}_{n}\right)$, then

$$
\begin{gathered}
\int_{\mathbf{X}} \pi(F)(\mathbf{x}, \mathbf{y}) d \mathbf{x}=\int_{\mathbf{X}} \pi(F)(\mathbf{x}, \mathbf{y}) d \mathbf{y}=\int_{\mathbb{H}_{n}} F(x, y, t) d x d y d t, \\
\pi\left(F_{S}\right)(\mathbf{x}, \mathbf{y})=s^{-(n+2) / 2} \pi(F)\left(\delta_{\left.\left.s^{-1 / 2} \mathbf{x}, \delta_{s^{-1 / 2}} \mathbf{y}\right)\right),}\right.
\end{gathered}
$$

where here and subsequently $F_{s}(x, y, t)=s^{-D / 2} F\left(\delta_{s^{-1 / 2}}(x, y, t)\right)$.

Further, for suitable functions $F$ on $\mathbb{H}_{n}$ one has

$$
\pi\left(X_{k}\right)_{\mathbf{x}} \pi(F)(\mathbf{x}, \mathbf{y})=-\pi\left(Y_{k} F\right)(\mathbf{x}, \mathbf{y}), \quad \pi\left(X_{k}\right)_{\mathbf{y}} \pi(F)(\mathbf{x}, \mathbf{y})=\pi\left(X_{k} F\right)(\mathbf{x}, \mathbf{y})(
$$

Lemma 2.1 There is a constant $C_{1}>0$ such that if $F \in L^{1}\left(\mathbb{H}_{n}\right)$, supp $F \subset$ $B_{\mathbb{H}_{n}}((0,0,0), R) \subset \mathbb{H}_{n}$, then

$$
\pi(F)(\mathbf{x}, \mathbf{y})=0 \text { for } d(\mathbf{x}, \mathbf{y})>C_{1} R
$$

Here $B_{\mathbb{H}_{n}}((0,0,0), R)=\left\{(x, y, t) \in \mathbb{H}_{n}:|(x, y, t)|<R\right\}$ and $|(x, y, t)|$ is the homogeneous norm in $\mathbb{H}_{n}$. 
Proof In the proof we will frequently use, without any comment, the formulas (2.1), (2.6), and (1.1). Assume that $d(\mathbf{x}, \mathbf{y})>C_{1} R$ with $C_{1}$ being large. If $\left|x^{\prime}-y^{\prime}\right|>R$, then $\pi(F)(\mathbf{x}, \mathbf{y})=0$. Thus for the remaining part of the proof we assume that $\left|x^{\prime}-y^{\prime}\right| \leq R$. We shall consider two cases.

Case 1: $\left|x^{\prime \prime}-y^{\prime \prime}\right|^{1 / 2} \leq\left|x^{\prime}\right|+\left|y^{\prime}\right|$. Then $\left|x^{\prime \prime}-y^{\prime \prime}\right|>C R\left(\left|x^{\prime}\right|+\left|y^{\prime}\right|\right)$, where $C$ is large if $C_{1}$ is chosen to be large. Consequently, $\left|x^{\prime \prime}-y^{\prime \prime}\right|>(C R)^{2}$. Hence, for $|z|<R$ we have

$$
\begin{aligned}
\left|x^{\prime \prime}-y^{\prime \prime}-\frac{1}{2} z \cdot\left(y^{\prime}+x^{\prime}\right)\right| & \geq\left|x^{\prime \prime}-y^{\prime \prime}\right|-R\left(\left|y^{\prime}\right|+\left|x^{\prime}\right|\right) \\
& \geq\left|x^{\prime \prime}-y^{\prime \prime}\right|\left(1-\frac{1}{C}\right) \geq(C R)^{2}\left(1-\frac{1}{C}\right) .
\end{aligned}
$$

Thus $\pi(F)(\mathbf{x}, \mathbf{y})=0$ if $C_{1}$ is large and, consequently, so is $C$.

Case 2: $\left|x^{\prime \prime}-y^{\prime \prime}\right|^{1 / 2}>\left|x^{\prime}\right|+\left|y^{\prime}\right|$. Then $\left|x^{\prime \prime}-y^{\prime \prime}\right|^{1 / 2}>C R$ and, again $C$ is large if $C_{1}$ is chosen to be large. For $|z|<R$ we have $\left|\frac{1}{2} z \cdot\left(y^{\prime}+x^{\prime}\right)\right|<R\left(\left|x^{\prime}\right|+\left|y^{\prime}\right|\right) \leq R\left|x^{\prime \prime}-y^{\prime \prime}\right|^{1 / 2}$. Therefore,

$$
\begin{aligned}
\left|x^{\prime \prime}-y^{\prime \prime}-\frac{1}{2} z \cdot\left(y^{\prime}+x^{\prime}\right)\right| & \geq\left|x^{\prime \prime}-y^{\prime \prime}\right|-R\left|x^{\prime \prime}-y^{\prime \prime}\right|^{1 / 2} \\
& =\left|x^{\prime \prime}-y^{\prime \prime}\right|^{1 / 2}\left(\left|x^{\prime \prime}-y^{\prime \prime}\right|^{1 / 2}-R\right) \\
& \geq C R(C R-R)=C(C-1) R^{2}
\end{aligned}
$$

which implies $\pi(F)(\mathbf{x}, \mathbf{y})=0$ if $C_{1}$ is large enough.

Lemma 2.2 There is a constant $C_{2}>0$ such that if $F$ is a bounded function on $\mathbb{H}_{n}$, supp $F \subset B((0,0,0), R) \subset \mathbb{H}_{n}$, then

$$
|\pi(F)(\mathbf{x}, \mathbf{y})| \leq C_{2} R^{D}|B(\mathbf{x}, R)|^{-1}\|F\|_{L^{\infty}\left(\mathbb{H}_{n}\right)} .
$$

Proof It suffices to prove the lemma for $F$ being the characteristic function of the ball $B_{\mathbb{H}_{n}}((0,0,0), R)$ for every $R>0$. Then, by (2.6),

$$
\begin{aligned}
|\pi(F)(\mathbf{x}, \mathbf{y})| \leq & \int_{\mathbb{R}^{n}} \chi_{[-R, R]^{n}}(z) \chi_{[-R, R]^{n}}\left(y^{\prime}-x^{\prime}\right) \chi_{\left[-R^{2}, R^{2}\right]} \\
& \times\left(y^{\prime \prime}-x^{\prime \prime}-\frac{1}{2} z \cdot\left(x^{\prime}+y^{\prime}\right)\right) d z .
\end{aligned}
$$

Assume that $\pi(F)(\mathbf{x}, \mathbf{y})>0$. We consider two cases.

Case 1: $R>\left|x^{\prime}\right| / C$, where $C>0$ is a large constant. Then, by $(1.3),|B(\mathbf{x}, R)| \sim$ $R^{n+2}$ and, consequently, $|\pi(F)(\mathbf{x}, \mathbf{y})| \leq 2 C R^{n} \sim R^{D}|B(\mathbf{x}, R)|^{-1}$.

Case 2: $R \leq\left|x^{\prime}\right| / C$. Notice that $\left|x^{\prime}+y^{\prime}\right| \sim\left|x^{\prime}\right|+\left|y^{\prime}\right| \sim\left|x^{\prime}\right|$, since $\left|x^{\prime}-y^{\prime}\right|<R$ and $C>0$ is large. Hence, by (2.11), 


$$
|\pi(F)(\mathbf{x}, \mathbf{y})| \lesssim R^{n-1} \frac{R^{2}}{\left|x^{\prime}+y^{\prime}\right|} \sim \frac{R^{n+1}}{\left|x^{\prime}\right|} \sim \frac{R^{D}}{|B(\mathbf{x}, R)|}
$$

\subsection{Heat kernel for $\mathcal{L}$}

The kernels of the semigroups $e^{-s \mathcal{L}}$ and $e^{-s L}$ are related by

$$
H_{s}(\mathbf{x}, \mathbf{y})=\pi\left(h_{s}\right)(\mathbf{x}, \mathbf{y}) .
$$

Let us also note that thanks to the homogeneity of $\mathcal{L}$ one has

$$
H_{S}(\mathbf{x}, \mathbf{y})=s^{-(n+2) / 2} H_{1}\left(\delta_{s^{-1 / 2}} \mathbf{x}, \delta_{s^{-1 / 2}} \mathbf{y}\right) .
$$

Proposition 2.3 (Gaussian bounds for $H_{S}$ ) There are constants $c, C>0$ such that

$$
H_{S}(\mathbf{x}, \mathbf{y}) \leq \frac{C}{|B(\mathbf{x}, \sqrt{s})|} e^{-c d(\mathbf{x}, \mathbf{y})^{2} / s} .
$$

Proof The proposition is well-known. For the convenience of the reader we present a short proof based on estimates of the heat kernel for the sub-Laplacian $L$ on the Heisenberg group combined with Lemmas 2.1 and 2.2. To this end from (2.4) we have

$$
\begin{aligned}
0 \leq h_{s}(\boldsymbol{x}) & \lesssim s^{-D / 2} \sum_{k=1}^{\infty} e^{-\alpha k^{2}} \chi_{B_{\mathbb{H}_{n}}(0, k)}\left(\delta_{s^{-1 / 2}} \boldsymbol{x}\right) \\
& =s^{-D / 2} \sum_{k=1}^{\infty} e^{-\alpha k^{2}} \chi_{B_{\mathbb{H}_{n}}(0, \sqrt{s} k)}(\boldsymbol{x}) .
\end{aligned}
$$

Using (2.12), Lemmas 2.1 and 2.2, we obtain

$$
0 \leq H_{s}(\mathbf{x}, \mathbf{y}) \lesssim s^{-D / 2} \sum_{k=1}^{\infty}(\sqrt{s} k)^{D}|B(\mathbf{x}, \sqrt{s} k)|^{-1} e^{-\alpha k^{2}} \chi_{B\left(\mathbf{x}, C_{1} \sqrt{s} k\right)}(\mathbf{y})
$$

Applying (1.4), we get

$$
\begin{aligned}
0 \leq H_{s}(\mathbf{x}, \mathbf{y}) & \lesssim \sum_{k=1}^{\infty} k^{D} \frac{B(\mathbf{x}, \sqrt{s}) \mid}{|B(\mathbf{x}, \sqrt{s} k)|}|B(\mathbf{x}, \sqrt{s})|^{-1} e^{-\alpha k^{2}} \chi_{B\left(\mathbf{x}, C_{1} \sqrt{s} k\right)}(\mathbf{y}) \\
& \lesssim \sum_{k=1}^{\infty} k^{D-n-1}|B(\mathbf{x}, \sqrt{s})|^{-1} e^{-\alpha k^{2}} \chi_{B\left(\mathbf{x}, C_{1} \sqrt{s} k\right)}(\mathbf{y}) \\
& \lesssim|B(\mathbf{x}, \sqrt{s})|^{-1} e^{-c d(\mathbf{x}, \mathbf{y})^{2} / s} .
\end{aligned}
$$


Lemma 2.4 There is a constant $C>0$ such that

$$
\left|H_{S}(\mathbf{x}, \mathbf{y})-H_{S}(\mathbf{z}, \mathbf{y})\right| \leq \frac{C}{|B(\mathbf{y}, \sqrt{s})|} \frac{d(\mathbf{x}, \mathbf{z})}{\sqrt{s}} .
$$

Proof Fix $\mathbf{y} \in \mathbf{X}$ and $s>0$ and set $F(\mathbf{x})=H_{S}(\mathbf{x}, \mathbf{y})$. Now, using (2.5) and the same arguments we have used in the proof of (2.13), we obtain

$$
\sum_{j=1}^{n}\left|\partial_{x_{j}^{\prime}} F(\mathbf{x})\right|^{2}+\left|x_{j}^{\prime} \partial_{x^{\prime \prime}} F(\mathbf{x})\right|^{2} \leq \frac{C}{s|B(\mathbf{y}, \sqrt{s})|^{2}}
$$

Finally, by the definition of the distance $d$, we have

$$
|F(\mathbf{x})-F(\mathbf{z})| \leq \frac{C d(\mathbf{x}, \mathbf{z})}{\sqrt{s}|B(\mathbf{y}, \sqrt{s})|}
$$

Corollary 2.5 (Hölder-type estimates for $H_{t}$ ) For $0<\gamma<1$ there are constants C, $c_{0}>0$ such that

$$
\left|H_{s}(\mathbf{x}, \mathbf{y})-H_{s}(\mathbf{x}, \mathbf{z})\right| \leq \frac{C}{|B(\mathbf{x}, \sqrt{s})|}\left(\frac{d(\mathbf{y}, \mathbf{z})}{\sqrt{s}}\right)^{\gamma}
$$

with the improvement

$$
\begin{aligned}
& \left|H_{S}(\mathbf{x}, \mathbf{y})-H_{S}(\mathbf{x}, \mathbf{z})\right| \leq \frac{C}{|B(\mathbf{x}, \sqrt{s})|}\left(\frac{d(\mathbf{y}, \mathbf{z})}{\sqrt{s}}\right)^{\gamma} e^{-c_{0} d(\mathbf{x}, \mathbf{y})^{2} / s} \\
& \quad \text { if } d(\mathbf{y}, \mathbf{z}) \leq d(\mathbf{x}, \mathbf{y}) / 2 .
\end{aligned}
$$

Lemma 2.6 (On diagonal lower bound of $H_{S}$ ) There is a constant $C>0$ such that

$$
H_{s}(\mathbf{x}, \mathbf{x}) \geq C|B(\mathbf{x}, \sqrt{s})|^{-1} \text { for every } \mathbf{x} \in \mathbf{X} \text {. }
$$

Proof By the homogeneity it suffices to prove the estimate for $s=1$. To this end

$$
H_{1}(\mathbf{x}, \mathbf{x}) \gtrsim \int_{\mathbb{R}^{n}} e^{-C|z|^{2}} e^{-C\left|z \cdot x^{\prime}\right|} d z \gtrsim \frac{1}{1+\left|x^{\prime}\right|} \sim|B(\mathbf{x}, 1)|^{-1} .
$$

\section{Proof of Theorem 1.1}

Proof To prove the theorem we use Uchiyama's results [25]. For this purpose we set

$$
\rho(\mathbf{x}, \mathbf{y})=\inf |B|
$$


where the infimum is taken over all closed balls $B$ containing $\mathbf{x}$ and $\mathbf{y}$. Then $\rho$ is a quasi-distance such that $\rho(\mathbf{x}, \mathbf{y}) \asymp|B(\mathbf{x}, d(\mathbf{x}, \mathbf{y}))|$ for all $\mathbf{x}, \mathbf{y} \in \mathbf{X}$ and

$$
\left|B_{\rho}(\mathbf{x}, r)\right| \asymp r \quad \text { for every } \mathbf{x} \in \mathbf{X} \text { and } r>0 \text {, }
$$

where $B_{\rho}(\mathbf{x}, r)$ denotes the closed quasi-ball with center $\mathbf{x}$ and radius $r$ (see, e.g. [1, Lemma 6.4] for the proof).

Define the new kernel $K_{r}(\mathbf{x}, \mathbf{y})$ by

$$
K_{r}(\mathbf{x}, \mathbf{y})=H_{t}(\mathbf{x}, \mathbf{y}),
$$

where $r=|B(\mathbf{x}, \sqrt{t})|$. The kernel $K_{r}(\mathbf{x}, \mathbf{y})$ satisfies the following assumptions of Uchiyama's theorem, which are stated in conditions (3.3)-(3.5) below.

- The on-diagonal lower estimate:

$$
K_{r}(\mathbf{x}, \mathbf{x}) \geq \frac{1}{A r}
$$

- Upper estimate: for every $\delta>0$,

$$
K_{r}(\mathbf{x}, \mathbf{y}) \leq \frac{A}{r}\left(1+\frac{\rho(\mathbf{x}, \mathbf{y})}{r}\right)^{-1-\delta}
$$

- Hölder estimate: there exist $C_{3}>0, \delta>0$, such that

$$
\begin{aligned}
\left|K_{r}(\mathbf{x}, \mathbf{y})-K_{r}(\mathbf{x}, \mathbf{z})\right| & \leq \frac{A}{r}\left(1+\frac{\rho(\mathbf{x}, \mathbf{y})}{r}\right)^{-1-2 \delta}\left(\frac{\rho(\mathbf{y}, \mathbf{z})}{r}\right)^{\delta} \text { if } \rho(\mathbf{y}, \mathbf{z}) \\
& \leq C_{3} \max \{r, \rho(\mathbf{x}, \mathbf{y})\} .
\end{aligned}
$$

The estimates (3.3)-(3.5) are consequences of (1.4), (2.21), (2.13), and Corollary 2.5 (see, e.g., [1, Appendix 3]).

Now we define the Hardy spaces $H_{\max , K_{r}}^{1}$ as the set of all $L^{1}\left(\mathbb{R}^{n+1}\right)$-functions $f$ such that $\left\|f^{(+)}\right\|_{L^{1}(\mathbf{X})}<\infty$, where $f^{(+)}=\sup _{r>0}\left|\int K_{r}(\mathbf{x}, \mathbf{y}) f(\mathbf{y}) d \mathbf{y}\right|$.

The atomic Hardy space $H_{\text {atom, } \infty}^{1}(\mathbf{X}, \rho)$ is defined in the standard way. A function $a$ is called an atom for $H_{\text {atom, } \infty}^{1}(\mathbf{X}, \rho)$, if there is a ball $B_{\rho}\left(\mathbf{x}_{0}, r\right)$ such that supp $a \subset$ $B_{\rho}\left(\mathbf{x}_{0}, r\right),\|a\|_{L^{\infty}} \leq\left|B_{\rho}\left(\mathbf{x}_{0}, r\right)\right|^{-1} \sim r^{-1}, \int a=0$. Now a function $f$ is an element of $H_{\text {atom, } \infty}^{1}(\mathbf{X}, \rho)$ if $f(\mathbf{x})=\sum_{k} \lambda_{k} a_{k}(\mathbf{x})$, where $a_{k}(\mathbf{x})$ are atoms for $H_{\text {atom, } \infty}^{1}(\mathbf{X}, \rho)$ and $\lambda_{k} \in \mathbb{C}$ with $\sum_{k}\left|\lambda_{k}\right|<\infty$. For such $f$ we set $\|f\|_{H_{\text {atom }, \infty}^{1}(\mathbf{X}, \rho)}=\inf \sum_{k}\left|\lambda_{k}\right|$, where infimum is taken over all such representations.

We are now in a position to state the following theorem of Uchiyama about atomic and maximal characterizations of Hardy spaces on a space of homogeneous type.

Theorem 3.1 [25, Corollary 1'] Assume that $\rho(\mathbf{x}, \mathbf{y})$ and $K_{r}(\mathbf{x}, \mathbf{y})$ satisfy (3.1) and (3.3)-(3.5). Then the spaces $H_{\max , K_{r}}^{1}$ and $H_{\text {atom, } \infty}^{1}(\mathbf{X}, \rho)$ coincide and the norms $\left\|f^{(+)}\right\|_{L^{1}(\mathbf{X})}$ and $\|f\|_{H_{\text {atom }, \infty}^{1}(\mathbf{X}, \rho)}$ are equivalent. 

then

It is easy to prove that there exists a constant $c \geq 1$ such that if $r=|B(\mathbf{x}, \sqrt{t})|$,

$$
B(\mathbf{x}, \sqrt{t}) \subset B_{\rho}(\mathbf{x}, r) \subset B(\mathbf{x}, c \sqrt{t}) .
$$

The above inclusions imply that the atomic Hardy spaces for $d(\mathbf{x}, \mathbf{y})$ and $\rho(\mathbf{x}, \mathbf{y})$ coincide. Moreover, the maximal functions for the kernels $K_{r}$ and $H_{t}$ are equal. Hence, Theorem 1.1 follows from Theorem 3.1.

\section{Farther Properties of $\pi$}

\subsection{Homogeneous Kernels}

A tempered distribution $S$ on $\mathbb{H}_{n}$ is said to be a regular kernel of order $r \in \mathbb{R}$ if $S$ coincides with a $C^{\infty}$ function $m(\boldsymbol{x})$ away from the origin and satisfies

$$
\left\langle S, f \circ \delta_{s}\right\rangle=s^{r}\langle S, f\rangle
$$

for any $f \in \mathcal{S}(\mathbb{H})$. Any regular kernel of order $r$ gives rise to the convolution operator

$$
f \mapsto f * \check{S}(\boldsymbol{x})=\left\langle S, f_{\boldsymbol{x}}\right\rangle, \quad f_{\boldsymbol{x}}(\boldsymbol{y})=f(\boldsymbol{x} \boldsymbol{y})
$$

which will be denoted by the same symbol $S$.

Any tempered distribution $S$ on $\mathbb{H}_{n}$ which is a regular kernel of order 0 is of the form

$$
\begin{aligned}
\langle S, f\rangle & =c_{1} f(0)+\lim _{\varepsilon \rightarrow 0} \int_{\varepsilon}^{\infty} \int_{\Sigma} m(\overline{\boldsymbol{x}}) f\left(\delta_{r} \overline{\boldsymbol{x}}\right) d \sigma(\overline{\boldsymbol{x}}) \frac{d r}{r} \\
& =c_{1} f(0)+\lim _{\varepsilon \rightarrow 0} \int_{|\boldsymbol{x}|>\varepsilon} m(\boldsymbol{x}) f(\boldsymbol{x}) d \boldsymbol{x},
\end{aligned}
$$

where $m$ is a $C^{\infty}$ function away from the origin, $m\left(\delta_{s} \boldsymbol{x}\right)$ $=s^{-D} m(\boldsymbol{x}), \int_{\Sigma} m(\overline{\boldsymbol{x}}) d \sigma(\overline{\boldsymbol{x}})=0$ (see [3, Lemma 2.4]). Here $\Sigma=\left\{\overline{\boldsymbol{x}} \in \mathbb{H}_{n}\right.$ : $|\overline{\boldsymbol{x}}|=1\}$ is the unit sphere in $\mathbb{H}_{n}$ and $d \sigma(\overline{\boldsymbol{x}})$ is the Radon measure on $\Sigma$ such that $\int_{\mathbb{H}_{n}} f(\boldsymbol{x}) d \boldsymbol{x}=\int_{0}^{\infty} \int_{\Sigma} f\left(\delta_{s} \overline{\boldsymbol{x}}\right) s^{D-1} d \sigma(\overline{\boldsymbol{x}}) d s$ (see [12, Proposition 1.5]).

Let $\varphi$ be a $C^{\infty}$ function on $\mathbb{H}_{n}$ such that $0 \leq \varphi \leq 1, \varphi(\boldsymbol{x})=\varphi(\boldsymbol{y})$ whenever $|\boldsymbol{x}|=|\boldsymbol{y}|, \operatorname{supp} \varphi \subset\left\{\boldsymbol{x} \in \mathbb{H}_{n}: \frac{1}{2} \leq|\boldsymbol{x}| \leq 2\right\}, \sum_{j=-\infty}^{\infty} \varphi\left(\delta_{2^{j}} \boldsymbol{x}\right)=1$ for $\boldsymbol{x} \neq 0$. Then any regular kernel $S$ of order 0 can be written as

$$
\langle S, f\rangle=c_{1} f(0)+\sum_{j=-\infty}^{\infty} \int_{\mathbb{H}_{n}} m_{j}(\boldsymbol{x}) f(\boldsymbol{x}) d \boldsymbol{x},
$$

where $m_{j}(\boldsymbol{x})=\varphi\left(\delta_{2^{j}} \boldsymbol{x}\right) m(\boldsymbol{x})$. Clearly, 


$$
\begin{aligned}
\left|X^{I} Y^{J} m_{j}(\boldsymbol{x})\right| & \leq C 2^{j(D+d(I)+d(J))}\left\|X^{I} Y^{J} m_{0}\right\|_{L^{\infty}}, \\
\int_{\mathbb{H}_{n}} m_{j}(\boldsymbol{x}) d \boldsymbol{x} & =0, \quad \int_{\mathbb{H}_{n}} X^{J} m_{j}(\boldsymbol{x}) d \boldsymbol{x}=0, \int_{\mathbb{H}_{n}} Y^{J} m_{j}(\boldsymbol{x}) d \boldsymbol{x}=0 .
\end{aligned}
$$

Let $m_{j}^{*}(\boldsymbol{x})=\overline{m_{j}\left(\boldsymbol{x}^{-1}\right)}=\overline{m_{j}(-\boldsymbol{x})}, \check{m}_{j}(\boldsymbol{x})=m_{j}(-\boldsymbol{x})$. It is not difficult to check that there are constants $C, c>0$ such that

$$
\left\|\left(m_{j}^{*} * m_{k}\right)^{\llcorner}\right\|_{L^{1}\left(\mathbb{H}_{n}\right)}+\left\|\left(m_{j} * m_{k}^{*}\right)^{\llcorner}\right\|_{L^{1}\left(\mathbb{H}_{n}\right)} \leq C 2^{-c|j-k|}\|m \varphi\|_{C^{1}}
$$

Thus, by the Cotlar-Stein lemma, $S f=f * \check{S}=c_{1} f+\sum_{j=-\infty}^{\infty} f * \check{m}_{j}$ defines a bounded operator on $L^{2}\left(\mathbb{H}_{n}\right)$ and, then on $L^{p}\left(\mathbb{H}_{n}\right), 1<p<\infty$, since $S$ is a CalderónZygmund operator on $\mathbb{H}_{n}$. Moreover, $\|S f\|_{L^{p}\left(\mathbb{H}_{n}\right)} \leq\left(C_{p}\|m \varphi\|_{C^{1}}+c_{1}\right)\|f\|_{L^{p}\left(\mathbb{H}_{n}\right)}$. The space of convolution operators with regular kernels of order 0 is an algebra with involution. Clearly,

$$
\int_{\mathbb{H}_{n}} S f \cdot \bar{g}=\int_{\mathbb{H}_{n}} f \cdot \overline{S^{*} g}, \quad \text { where }\left\langle S^{*}, f\right\rangle=\bar{c}_{1} f(0)+\mathrm{pv} \int_{\mathbb{H}_{n}} m^{*}(\boldsymbol{x}) f(\boldsymbol{x}) d \boldsymbol{x} .
$$

Set $\pi(S) f=c_{1} f+\sum_{j=-\infty}^{\infty} \pi\left(m_{j}\right) f$. Then using the Coifman-Weiss transference principle [9] we have $\|\pi(S)\|_{L^{2}(\mathbf{X}) \rightarrow L^{2}(\mathbf{X})} \leq C\|S\|_{L^{2}\left(\mathbb{H}_{n}\right) \rightarrow L^{2}\left(\mathbb{H}_{n}\right)}$. Moreover, from (2.7), (2.9), (4.2), Lemmas 2.1 and 2.2 we conclude

$$
\begin{gathered}
\int_{\mathbf{X}} \pi\left(m_{j}\right)(\mathbf{x}, \mathbf{y}) d \mathbf{x}=\int_{\mathbf{X}} \pi\left(m_{j}\right)(\mathbf{x}, \mathbf{y}) d \mathbf{y}=0 \\
\pi\left(m_{j}\right)(\mathbf{x}, \mathbf{y})=0 \text { for } d(\mathbf{x}, \mathbf{y})>2 C_{1} 2^{-j} \\
\left|\pi\left(X^{I}\right)_{\mathbf{x}} \pi\left(X^{J}\right)_{\mathbf{y}} \pi\left(m_{j}\right)(\mathbf{x}, \mathbf{y})\right| \leq C\left\|X^{J} Y^{I} m_{0}\right\|_{L^{\infty}} 2^{(d(I)+d(J)) j}\left|B\left(\mathbf{x}, 2^{-j}\right)\right|^{-1}, \\
\pi\left(m_{j}^{*}\right)(\mathbf{x}, \mathbf{y})=\frac{\pi\left(m_{j}\right)(\mathbf{y}, \mathbf{x})}{}
\end{gathered}
$$

Consequently, $\pi(S)$ is a Calderón-Zygmund operator with the associated kernel

$$
k(\mathbf{x}, \mathbf{y})=\sum_{j=-\infty}^{\infty} \pi\left(m_{j}\right)(\mathbf{x}, \mathbf{y})
$$

which satisfies

$$
\pi(S) f(\mathbf{x})=\int k(\mathbf{x}, \mathbf{y}) f(\mathbf{y}) d \mathbf{y} \text { for } \mathbf{x} \notin \operatorname{supp} f,
$$




$$
\left|\pi\left(X^{I}\right)_{\mathbf{x}} \pi\left(X^{J}\right)_{\mathbf{y}} k(\mathbf{x}, \mathbf{y})\right| \leq C_{I, J}\left\|X^{J} Y^{I} m_{0}\right\|_{L^{\infty}}|B(\mathbf{x}, d(\mathbf{x}, \mathbf{y}))|^{-1} d(\mathbf{x}, \mathbf{y})^{-d(I)-d(J)},
$$

$$
k\left(\delta_{t} \mathbf{x}, \delta_{t} \mathbf{y}\right)=t^{-(n+2)} k(\mathbf{x}, \mathbf{y}) .
$$

Lemma 4.1 There is a constant $C>0$ such that for any regular kernel $S$ of order 0 on $\mathbb{H}_{n}$ and for every function $f \in C_{c}^{1}\left(B\left(\mathbf{x}_{0}, r_{0}\right)\right)$ we have

$$
|\pi(S) f(\mathbf{x})| \leq\left(2 c_{1}+C\left\|m_{0}\right\|_{L^{\infty}}\right) r_{0} \sum_{k=1}^{2 n}\left\|\pi\left(X_{k}\right) f\right\|_{L^{\infty}} .
$$

Proof Note that (1.2) and (4.5) imply

$$
\|f\|_{L^{\infty}} \leq 2 r_{0} \sum_{k=1}^{2 n}\left\|\pi\left(X_{k}\right) f\right\|_{L^{\infty}}
$$

and

$$
\left|\pi\left(m_{j}\right)(\mathbf{x}, \mathbf{y})\right| \leq C\left|B\left(\mathbf{x}, 2^{-j}\right)\right|^{-1}\left\|m_{0}\right\|_{L^{\infty}} .
$$

Let $j_{0}$ be such that $2^{-j_{0}}<r_{0} \leq 2^{-j_{0}+1}$. Clearly, by (4.3) and (4.4), we get

$$
\begin{aligned}
& \left|\sum_{j=-\infty}^{\infty} \int \pi\left(m_{j}\right)(\mathbf{x}, \mathbf{y}) f(\mathbf{y}) d \mathbf{y}\right| \\
& \leq \sum_{j<j_{0}} \int_{B\left(\mathbf{x}_{0}, r_{0}\right) \cap B\left(\mathbf{x}, C_{1} 2^{-j+1}\right)}\left|\pi\left(m_{j}\right)(\mathbf{x}, \mathbf{y})\right||f(\mathbf{y})| d \mathbf{y} \\
& \quad+\sum_{j \geq j_{0}} \int_{B\left(\mathbf{x}, C_{1} 2^{-j+1}\right)}\left|\pi\left(m_{j}\right)(\mathbf{x}, \mathbf{y})\right||f(\mathbf{y})-f(\mathbf{x})| d \mathbf{y} \\
& =J_{1}+J_{2} .
\end{aligned}
$$

Observe that if $B\left(\mathbf{x}_{0}, r_{0}\right) \cap B\left(\mathbf{x}, C_{1} 2^{-j+1}\right) \neq \emptyset$ with $j<j_{0}$, then $\left|B\left(\mathbf{x}, 2^{-j}\right)\right| \sim$ $\left|B\left(\mathbf{x}_{0}, 2^{-j}\right)\right|$. Hence, applying (4.10), (4.11) and (1.4) we obtain

$$
\begin{aligned}
J_{1} & \leq \sum_{j<j_{0}}\left\|m_{0}\right\|_{L^{\infty}}\left|B\left(\mathbf{x}, 2^{-j}\right)\right|^{-1} 2 r_{0}\left(\sum_{k=1}^{2 n}\left\|\pi\left(X_{k}\right) f\right\|_{L^{\infty}}\right)\left|B\left(\mathbf{x}_{0}, r_{0}\right)\right| \\
& \leq C r_{0}\left\|m_{0}\right\|_{L^{\infty}}\left(\sum_{k=1}^{2 n}\left\|\pi\left(X_{k}\right) f\right\|_{L^{\infty}}\right) \sum_{j<j_{0}}\left(\frac{r_{0}}{2^{-j}}\right)^{n+1} \\
& \leq C r_{0}\left\|m_{0}\right\|_{L^{\infty}} \sum_{k=1}^{2 n}\left\|\pi\left(X_{k}\right) f\right\|_{L^{\infty}}
\end{aligned}
$$


To estimate $J_{2}$ we use (1.2) together with (4.11) and get

$$
\begin{aligned}
J_{2} & \leq C \sum_{j \geq j_{0}}\left\|m_{0}\right\|_{L^{\infty}}\left(\sum_{k=1}^{2 n}\left\|\pi\left(X_{k}\right) f\right\|_{L^{\infty}}\right) 2^{-j} \\
& \leq C r_{0}\left\|m_{0}\right\|_{L^{\infty}} \sum_{j=1}^{2 n}\left\|\pi\left(X_{j}\right) f\right\|_{L^{\infty}} .
\end{aligned}
$$

Thanks to Lemma 4.1 for $f \in L^{1}(\mathbf{X})$ and a regular kernel $S$ of order 0 we define $\pi(S) f$ in the sense of distribution setting

$$
\langle\pi(S) f, \varphi\rangle=\langle f, \pi(\check{S}) \varphi\rangle, \varphi \in C_{c}^{\infty}\left(\mathbb{R}^{n+1}\right) .
$$

Lemma 4.2 There is a constant $C>0$ such that for any regular kernel $S$ of order 0 on $\mathbb{H}_{n}$ which has the form (4.1) we have

$$
\|\pi(S) f\|_{H_{\mathcal{L}}^{1}} \leq\left(c_{1}+C\left\|m_{0}\right\|_{C^{1}}\right)\|f\|_{H_{\mathcal{L}}^{1}}
$$

Proof The proof is standard. For the sake of completeness we present its sketch. Without loss of generality we can assume that $c_{1}=0$. Because $\pi(S)$ maps continuously $L^{1}(\mathbf{X})$ to $\mathcal{D}^{\prime}\left(\mathbb{R}^{n+1}\right)$, it suffices to prove that there is a constant $C>0$ such that $\|\pi(S) a\|_{H_{\mathcal{L}}^{1}} \leq C$ for every atom $a \in H_{\text {atom, } \infty}^{1}(\mathbf{X})$. Fix a $(1, \infty)$-atom $a$ associated with a ball $B\left(\mathbf{y}_{0}, r_{0}\right)$. Since $\sum_{j} \pi\left(m_{j}\right) a$ converges in $L^{2}(\mathbf{X})$, it converges in the $L^{1}(\mathbf{X})$ norm on $B\left(\mathbf{y}_{0}, 2 r_{0}\right)$ as well. Note also that $\pi\left(m_{j}\right) a(\mathbf{x})=0$ for $d\left(\mathbf{x}, \mathbf{y}_{0}\right)>2 C_{1} 2^{-j}+r_{0}$. If $2 r_{0}<d\left(\mathbf{x}, \mathbf{y}_{0}\right) \leq 2 C_{1} 2^{-j}+r_{0}$, then applying (1.2) and (4.5) we get

$$
\begin{aligned}
\left|\pi\left(m_{j}\right) a(\mathbf{x})\right| & =\left|\int_{B\left(\mathbf{y}_{0}, r_{0}\right)}\left(\pi\left(m_{j}\right)(\mathbf{x}, \mathbf{y})-\pi\left(m_{j}\right)\left(\mathbf{x}, \mathbf{y}_{0}\right)\right) a(\mathbf{y}) d \mathbf{y}\right| \\
& \leq C\left\|m_{0}\right\|_{C^{1}} 2^{j} r_{0}\left|B\left(\mathbf{y}_{0}, 2^{-j}\right)\right|^{-1} .
\end{aligned}
$$

Hence, $\sum_{j} \pi\left(m_{j}\right) a$ converges in $L^{1}(\mathbf{X})$ and $L^{2}(\mathbf{X})$ to $\pi(S) a$ and $\int \pi(S) a(\mathbf{x}) d \mathbf{x}=$ 0 . Moreover,

$$
\begin{aligned}
|\pi(S) a(\mathbf{x})| & \leq C\left\|m_{0}\right\|_{C^{1}} \frac{r_{0}}{\left|B\left(\mathbf{y}_{0}, d\left(\mathbf{x}, \mathbf{y}_{0}\right)\right)\right| d\left(\mathbf{x}, \mathbf{y}_{0}\right)} \text { for } d\left(\mathbf{x}, \mathbf{y}_{0}\right)>2 r_{0}, \\
\|\pi(S) a\|_{L^{2}(\mathbf{X})} & \leq C\left(\left\|m_{0}\right\|_{C^{1}}+c_{1}\right)\left|B\left(\mathbf{y}_{0}, r_{0}\right)\right|^{-1 / 2} .
\end{aligned}
$$

So, $\pi(S) a$ can be written as $\pi(S) a=\sum_{j} \lambda_{j} a_{j}$ with $a_{j}$ being $(1,2)$-atoms and $\sum_{j}\left|\lambda_{j}\right| \leq C$.

Let $\psi$ be a Schwartz class function on $[0, \infty)$ and $d E_{L}$ and $d E_{\mathcal{L}}$ be the spectral measures for $L$ and $\mathcal{L}$ respectively. It is well known that the operator

$$
\psi(L) f=\int_{0}^{\infty} \psi(\lambda) d E_{L}(\lambda) f
$$


is a convolution operator with a Schwartz class function on $\mathbb{H}_{n}$ denoted by the same symbol $\psi(L)(\boldsymbol{x})$, that is, $\psi(L) f(\boldsymbol{x})=f * \psi(L)(\boldsymbol{x})$ (see, e.g., [14]). Moreover, for every multi-index $I$ and $M \geq 0$ there is $N>0$ and a constant $C_{I, M, N}>0$ such that

$$
\begin{aligned}
& \sup _{\boldsymbol{x} \in \mathbb{H}_{n}}(1+|\boldsymbol{x}|)^{M}\left(\left|X^{I} \psi(L)(\boldsymbol{x})\right|+\left|Y^{I} \psi(L)(\boldsymbol{x})\right|\right) \\
& \leq C_{I, M, N} \sup _{\lambda>0}(1+\lambda)^{N}\left(\sum_{j=0}^{N}\left|\psi^{(j)}(\lambda)\right|\right) .
\end{aligned}
$$

By homogeneity,

$$
\psi(t L)(\boldsymbol{x})=t^{-D / 2} \psi(L)\left(\delta_{t^{-1 / 2}} \boldsymbol{x}\right)
$$

Clearly, the operator

$$
\psi(\mathcal{L}) f=\int_{0}^{\infty} \psi(\lambda) d E_{\mathcal{L}}(\lambda) f
$$

is of the form

$$
\psi(\mathcal{L}) f(\mathbf{x})=\int_{\mathbf{X}} \psi(\mathcal{L})(\mathbf{x}, \mathbf{y}) f(\mathbf{y}) d \mathbf{y}
$$

where

$$
\psi(\mathcal{L})(\mathbf{x}, \mathbf{y})=\pi(\psi(L))(\mathbf{x}, \mathbf{y}) .
$$

For detailed spectral properties of $\mathcal{L}$ we refer the reader to [19].

\subsection{Riesz Transforms}

The Riesz transforms $R_{j}, j=1,2, \ldots, 2 n$, on the Heisenberg group $\mathbb{H}_{n}$ are defined by $R_{j} f=X_{j} L^{-1 / 2} f=\lim _{\varepsilon \rightarrow 0} c \int_{\varepsilon}^{\varepsilon^{-1}} X_{j} e^{-t L} f \frac{d t}{\sqrt{t}}=\lim _{\varepsilon \rightarrow 0} f *\left(c \int_{\varepsilon}^{\varepsilon^{-1}} X_{j} h_{t} \frac{d t}{\sqrt{t}}\right)$. By the Cotlar-Stein almost orthogonality principle the above limit defines a bounded operator on $L^{2}\left(\mathbb{H}_{n}\right)$. One can also prove that $R_{j}$ are the principal valued convolution singular integral operators $R_{j} f=f * \check{R}_{j}$, where $R_{j}(\boldsymbol{x})=-c \int_{0}^{\infty} Y_{j} h_{t}(\boldsymbol{x}) \frac{d t}{\sqrt{t}}$ are real-valued regular kernels of order 0 .

Similarly the Riesz transforms $\mathcal{R}_{j}$ associated with the Grushin operator are defined by $\mathcal{R}_{j}=c \pi\left(X_{j}\right) \mathcal{L}^{-1 / 2}, j=1,2, \ldots, 2 n$. Clearly, $\mathcal{R}_{j}=\pi\left(R_{j}\right)$. Thus $\mathcal{R}_{j}$ are Calderón-Zygmund operators on $\mathbf{X}$, which are bounded on $L^{p}(\mathbf{X}), 1<p<\infty$, and, by Lemma 4.2 , bounded on $H_{\mathcal{L}}^{1}$. For boundedness of $\mathcal{R}_{j}$ on $L^{p}(\mathbf{X})$ see also [16]. 


\section{$5(\star)$ Property and Decomposition of $B M O(X)$}

Let $\vec{S}=\left(S_{1}, S_{2}, \ldots, S_{d}\right)$ be a system of regular kernels of order 0 on $\mathbb{H}_{n}$. We say that it fulfills condition $(\star)$ if for every unit vector $v \in \mathbb{R}^{d}$ there are regular kernels $T_{j}$ of order zero, $\left\langle T_{j}, f\right\rangle=c_{j} f(0)+\mathrm{pv} \int_{\mathbb{H}_{n}} m^{\{j\}}(\boldsymbol{x}) f(\boldsymbol{x}) d \boldsymbol{x}, j=1,2, \ldots, d$, such that

$(\star)\left\{\begin{array}{l}\sum_{j=1}^{d} S_{j}^{*} T_{j}=I, \\ \sum_{j=1}^{d} v_{j} T_{j}=0, \\ \left|c_{j}\right| \leq C \text { with } C \text { independent of } v, \\ \left|X^{I} m^{\{j\}}(\boldsymbol{x})\right|+\left|Y^{I} m^{\{j\}}(\boldsymbol{x})\right| \leq C_{I} \text { for }|\boldsymbol{x}|=1, \text { with } C_{I} \text { independent of } v .\end{array}\right.$

Theorem 5.1 (Christ and Geller [5, Sect. 6]) The system of the regular kernels of order zero $\left\{\delta_{0}, R_{1}, R_{2}, \ldots, R_{2 n}\right\}$ on the Heisenberg group $\mathbb{H}_{n}$ fulfils condition ( $\star$ ).

A locally integrable function $f$ on $\mathbf{X}$ is said to be an element of $B M O(\mathbf{X})$ if

$$
\|f\|_{B M O(\mathbf{X})}:=\sup _{\mathbf{y} \in \mathbf{X}, r>0} \frac{1}{|B(\mathbf{y}, r)|} \int_{B(\mathbf{y}, r)}\left|f(\mathbf{x})-f_{B(\mathbf{x}, r)}\right| d \mathbf{x}<\infty
$$

here $f_{B(\mathbf{y}, r)}=|B(\mathbf{y}, r)|^{-1} \int_{B(\mathbf{y}, r)} f(\mathbf{x}) d \mathbf{x}$ denotes the mean value of $f$ over $B(\mathbf{y}, r)=$ $\{\mathbf{x} \in \mathbf{X}: d(\mathbf{y}, \mathbf{x})<r\}$.

Our goal of this section is to prove the following theorem.

Theorem 5.2 Assume that $S_{j}, j=1,2, \ldots, d$, is a system of operators satisfying ( $\star$. Then there is a constant $C>0$ such that any compactly supported BMO(X) function $f$ can be written as

$$
f=\sum_{j=1}^{d} \pi\left(S_{j}^{*}\right) \boldsymbol{g}_{j}+\boldsymbol{g}_{0}
$$

with

$$
\sum_{j=0}^{d}\left\|\boldsymbol{g}_{j}\right\|_{\infty} \leq C\|f\|_{B M O(\mathbf{X})}
$$

Moreover,

$$
\sum_{j=0}^{d}\left\|\boldsymbol{g}_{j}\right\|_{L^{2}(\mathbf{X})}<\infty
$$

For the proof of the theorem we follow methods presented in Christ and Geller [5] about decompositions of BMO functions on homogeneous Lie groups (see also 
the original Uchiyama's proof [26] of constructive Fefferman-Stein decomposition of BMO functions on the Euclidean spaces).

There is no loss of generality to assume that $\|f\|_{B M O(\mathbf{X})}=\varepsilon$ with $\varepsilon>0$ very small to be determined latter on.

Let us also emphasize that for any $t>0$ the mapping $f \mapsto f \circ \delta_{t}$ is an isometry on $L^{\infty}$ and $B M O(\mathbf{X})$.

The main step of the proof of Theorem 5.2 is the following theorem.

Theorem 5.3 Assume that $S_{j}, j=1,2, \ldots, d$, is a system of operators satisfying $(\star)$. Then there are constants constant $C_{8}, C_{9}, \varepsilon_{0}>0$ such that any $B M O(\mathbf{X})$ function $f$ supported in any ball $B\left(\mathbf{z}_{B}, r\right)$ with $\|f\|_{B M O(\mathbf{X})}=\varepsilon<\varepsilon_{0}$, can be written as

$$
\begin{gathered}
f=\sum_{j=1}^{d} \pi\left(S_{j}^{*}\right) \widetilde{g}_{j}+\widetilde{g}_{0}+f_{1}, \\
\sum_{j=1}^{d}\left\|\widetilde{g}_{j}\right\|_{\infty} \leq 3 d, \quad\left\|\widetilde{g}_{0}\right\|_{L^{\infty}} \leq C_{9}\|f\|_{B M O(\mathbf{X})}, \\
\left\|f_{1}\right\|_{B M O(\mathbf{X})} \leq C_{9} \varepsilon^{2}, \quad \operatorname{supp} f_{1} \subset B\left(\mathbf{z}_{B}, C_{8} r\right),
\end{gathered}
$$

Moreover,

$$
\sum_{j=0}^{d}\left\|\widetilde{g}_{j}\right\|_{L^{2}(\mathbf{X})} \leq C_{9}\left|B\left(\mathbf{z}_{B}, r\right)\right|^{1 / 2}\|f\|_{B M O(\mathbf{X})}
$$

For the proof of Theorem 5.3 we adapt arguments of Christ and Geller [5]. For the convenience of the reader we present all the details in Appendixes 1 and 2.

Proof of Theorem 5.2 Fix $0<\varepsilon<\varepsilon_{0}$ such that $C_{9} \varepsilon<1$ and $C_{9} C_{8}^{3 / 2} \varepsilon<1$. Decompose $f$ according to Theorem 5.3. If $f_{1}=0$ we are done. Otherwise we apply Theorem 5.3 to the function $\varepsilon\left\|f_{1}\right\|_{B M O(\mathbf{X})}^{-1} f_{1}$ and obtain functions $f_{2}, \widetilde{g}_{j}^{\{1\}}, j=0,1, \ldots, d$, such that

$$
\begin{gathered}
f_{1}=\sum_{j=1}^{d} \pi\left(S_{j}^{*}\right) \widetilde{g}_{j}^{\{1\}}+\widetilde{g}_{0}^{\{1\}}+f_{2}, \\
\sum_{j=1}^{d}\left\|\widetilde{g}_{j}^{\{1\}}\right\|_{L^{\infty}} \leq 3 d \frac{\left\|f_{1}\right\|_{B M O(\mathbf{X})}}{\varepsilon} \leq 3 d C_{9} \varepsilon, \\
\mid \widetilde{g}_{0}^{\{1\}}\left\|_{L^{\infty}} \leq C_{9}\right\| f_{1} \|_{B M O(\mathbf{X})} \leq C_{9}^{2} \varepsilon^{2}, \\
\left\|f_{2}\right\|_{B M O(\mathbf{X})} \leq \frac{\left\|f_{1}\right\|_{B M O(\mathbf{X})}}{\varepsilon} C_{9} \varepsilon^{2} \leq C_{9}^{2} \varepsilon^{3}, \quad \operatorname{supp} f_{2} \subset B\left(\mathbf{z}_{B}, C_{8}^{2} r\right),
\end{gathered}
$$




$$
\begin{aligned}
\sum_{j=0}^{d}\left\|\widetilde{g}_{j}^{\{1\}}\right\|_{L^{2}(\mathbf{X})} & \leq \frac{\left\|f_{1}\right\|_{B M O(\mathbf{X})}}{\varepsilon} C_{9}\left|B\left(\mathbf{z}_{B}, C_{1} r\right)\right|^{1 / 2} \varepsilon \\
& \leq C_{9}^{2}\left|B\left(\mathbf{z}_{B}, C_{8} r\right)\right|^{1 / 2} \varepsilon^{2} .
\end{aligned}
$$

Set $\widetilde{g}_{j}^{\{0\}}=\widetilde{g}_{j}, j=0,1, \ldots, d$. Continuing this procedure we obtain sequences of functions $\widetilde{g}_{j}^{\{n\}}, j=0,1, \ldots, d, n=0,1,2, \ldots$, and $f_{n}$ such that

$$
\begin{gathered}
f=\sum_{j=1}^{d} \pi\left(S_{j}^{*}\right) \widetilde{g}_{j}^{\{0\}}+\widetilde{g}_{0}^{\{0\}}+f_{1}, \\
f_{n}=\sum_{j=1}^{d} \pi\left(S_{j}^{*}\right) g_{j}^{\{n\}}+g_{0}^{\{n\}}+f_{n+1}, \\
\sum_{j=1}^{d}\left\|\widetilde{g}_{j}^{\{n\}}\right\|_{L^{\infty} \leq 3 d C_{9}^{n} \varepsilon^{n},} \sum_{j=0}^{d}\left\|\widetilde{g}_{j}^{\{n\}}\right\|_{L^{2}(\mathbf{X})} \leq C_{9}^{n+1}\left|B\left(\mathbf{z}_{B}, C_{8}^{n} r\right)\right|^{1 / 2} \varepsilon^{n+1}, \\
\left\|f_{n+1}\right\|_{B M O(\mathbf{X})} \leq C_{9}^{n+1} \varepsilon^{n+2}, \quad \operatorname{supp} f_{n+1} \subset B\left(\mathbf{z}_{B}, C_{8}^{n+1} r\right) .
\end{gathered}
$$

Observe that

$$
\begin{aligned}
&\left\|f_{n}\right\|_{L^{2}(\mathbf{X})} \leq C^{\prime}\left|B\left(\mathbf{z}_{B}, C_{1}^{n} r\right)\right|^{1 / 2}\left\|f_{n}\right\|_{B M O(\mathbf{X})} \\
& \leq C^{\prime \prime} C_{8}^{3 n / 2}\left|B\left(\mathbf{z}_{B}, r\right)\right|^{1 / 2} C_{2}^{n} \varepsilon^{n} \rightarrow 0, \quad \text { as } n \rightarrow \infty \\
& \sum_{n=0}^{\infty} \sum_{j=0}^{d}\left\|\widetilde{g}_{j}^{\{n\}}\right\|_{L^{2}(\mathbf{X})} \leq \sum_{n=0}^{\infty} C_{9}^{n+1} C_{8}^{3 n / 2}\left|B\left(\mathbf{z}_{B}, r\right)\right|^{1 / 2} \varepsilon^{n+1}
\end{aligned}
$$

Putting $\boldsymbol{g}_{j}=\sum_{n=0}^{\infty} \widetilde{g}_{j}^{\{n\}}$ we obtain Theorem 5.2.

\section{Proof of Theorem 1.2}

Let $V M O(\mathbf{X})$ be the closure of the space of continuous functions with compact support in the $B M O$-norm. It is well-known (see [8, Theorem 4.1]) that $V M O(\mathbf{X})$ is a predual space to $H_{\text {atom, } \infty}^{1}(\mathbf{X})$, that is, $V M O(\mathbf{X})^{*}=H_{\text {atom, } \infty}^{1}(\mathbf{X})$ in the sense that any functional $\Phi$ on $\operatorname{VMO}(\mathbf{X})$ is of the form $\Phi(f)=\int f(\mathbf{x}) \overline{F(\mathbf{x})} d \mathbf{x}$ for $f \in C_{c}(\mathbf{X})$, where $F \in H_{\text {atom, } \infty}^{1}(\mathbf{X})$.

Assume firstly that $F \in L^{1}(\mathbf{X}) \cap L^{2}(\mathbf{X})$ and $\mathcal{R}_{j} F \in L^{1}(\mathbf{X}), j=1,2, \ldots, 2 n$. If $f$ is compactly supported continuous function on $\mathbf{X}$, then, according to Theorems 
5.1 and 5.2, there are functions $\boldsymbol{g}_{j} \in L^{\infty}(\mathbf{X}) \cap L^{2}(\mathbf{X}), j=0,1,2, \ldots, 2 n$, such that $\sum_{j=0}^{2 n}\left\|\boldsymbol{g}_{j}\right\|_{\infty} \leq C\|f\|_{B M O(\mathbf{X})}$ and $f=\boldsymbol{g}_{0}+\sum_{j=1}^{2 n} \mathcal{R}_{j}^{*} \boldsymbol{g}_{j}$. Hence,

$$
\begin{aligned}
\left|\int_{\mathbf{X}} f(\mathbf{x}) \overline{F(\mathbf{x})} d \mathbf{x}\right| & =\left|\int_{\mathbf{X}}\left(\boldsymbol{g}_{0}+\sum_{j=1}^{2 n} \mathcal{R}_{j}^{*} \boldsymbol{g}_{j}\right) \bar{F} d \mathbf{x}\right| \\
& =\left|\int\left(\boldsymbol{g}_{0}(\mathbf{x}) \overline{F(\mathbf{x})}+\sum_{j=1}^{2 n} \boldsymbol{g}_{j}(\mathbf{x}) \overline{\mathcal{R}_{j} F(\mathbf{x})}\right) d \mathbf{x}\right| \\
& \leq\|f\|_{B M O(\mathbf{X})}\left(\|F\|_{L^{1}(\mathbf{X})}+\sum_{j=1}^{2 n}\left\|\mathcal{R}_{j} F\right\|_{L^{1}(\mathbf{X})}\right) .
\end{aligned}
$$

Thus, the integral $f \mapsto \int f(\mathbf{x}) \overline{F(\mathbf{x})} d \mathbf{x}$ has the unique extension to a bounded functional on $\operatorname{VMO}(\mathbf{X})$ and, consequently, $F \in H_{\text {atom, } \infty}^{1}(\mathbf{X})$ with

$$
\|F\|_{H_{\text {atom }, \infty}^{1}(\mathbf{X})} \leq C\left(\|F\|_{L^{1}(\mathbf{X})}+\sum_{j=1}^{2 n}\left\|\mathcal{R}_{j} F\right\|_{L^{1}(\mathbf{X})}\right) .
$$

We now relax the assumption $F \in L^{2}(\mathbf{X})$ assuming only that $F \in L^{1}(\mathbf{X})$ with $\mathcal{R}_{j} F \in L^{1}(\mathbf{X})$.

Lemma 6.1 Let $S$ be a regular kernel of order zero on $\mathbb{H}_{n}$. Then there is a constant $C>0$ such that for every $t>0$

$$
\left\|\left[\pi(S), e^{-t \mathcal{L}}\right] F\right\|_{L^{1}(\mathbf{X})} \leq C\|F\|_{L^{1}(\mathbf{X})},
$$

where $\left[\pi(S), e^{-t \mathcal{L}}\right]=\pi(S) e^{-t \mathcal{L}}-e^{-t \mathcal{L}} \pi(S)$ is the commutator of $\pi(S)$ and $e^{-t \mathcal{L}}$. We shall postpone the proof of the lemma to the end of the section.

Note that $e^{-t \mathcal{L}} F \in L^{2}(\mathbf{X})$ for $F \in L^{1}(\mathbf{X})$. Thus from Lemma 6.1 we conclude that

$$
\left\|\mathcal{R}_{j} e^{-t \mathcal{L}} F\right\|_{L^{1}(\mathbf{X})} \leq C\left(\left\|\mathcal{R}_{j} F\right\|_{L^{1}(\mathbf{X})}+\|F\|_{L^{1}(\mathbf{X})}\right)
$$

with a constant $C$ independent of $t>0$. The first part of the proof combined with (6.3) and Theorem 1.1 lead to

$$
\begin{aligned}
\left\|e^{-t \mathcal{L}} F\right\|_{H_{\mathcal{L}}^{1}} & \leq C\left(\sum_{j=1}^{2 n}\left\|\mathcal{R}_{j} e^{-t \mathcal{L}} F\right\|_{L^{1}(\mathbf{X})}+\left\|e^{-t \mathcal{L}} F\right\|_{L^{1}(\mathbf{X})}\right) \\
& \leq C\left(\sum_{j=1}^{2 n}\left\|\mathcal{R}_{j} F\right\|_{L^{1}(\mathbf{X})}+\|F\|_{L^{1}(\mathbf{X})}\right)
\end{aligned}
$$


because $e^{-t \mathcal{L}}$ is uniformly bounded on $L^{1}(\mathbf{X})$. Since $\mathcal{M}_{\mathcal{L}}\left(e^{-t_{1}} \mathcal{L} F\right)(\mathbf{x})$ $\leq \mathcal{M}_{\mathcal{L}}\left(e^{-t_{2}} \mathcal{L} F\right)(\mathbf{x})$ for $0<t_{2}<t_{1}$, we deduce from the Lebesgue monotone convergence theorem that $\mathcal{M}_{\mathcal{L}} F \in L^{1}(\mathbf{X})$ and

$$
\left\|\mathcal{M}_{\mathcal{L}} F\right\|_{L^{1}(\mathbf{X})} \leq C\left(\sum_{j=1}^{2 n}\left\|\mathcal{R}_{j} F\right\|+\|F\|_{L^{1}(\mathbf{X})}\right) .
$$

This completes the proof of the first inequality of (1.6).

The proof of the second inequality in (1.6) is standard and follows from the fact that $\mathcal{R}_{j}$ are Calderón-Zygmund operators (see Sect. 4). We omit the details.

Proof of Lemma 6.1 By the homogeneity it suffices to prove the lemma for $t=1$. Recall that

$$
\int_{\mathbf{X}} H_{t}(\mathbf{x}, \mathbf{z}) d \mathbf{z}=\int_{\mathbf{X}} H_{t}(\mathbf{z}, \mathbf{y}) d \mathbf{z}=1,
$$

where $H_{t}(\mathbf{x}, \mathbf{y})$ denote the integral kernel for $e^{-t \mathcal{L}}$. Let $m_{j}$ be as in Sect. 4.1. Set

$$
M_{j}=\int\left|\int\left(H_{1}(\mathbf{x}, \mathbf{z}) \pi\left(m_{j}\right)(\mathbf{z}, \mathbf{y})-\pi\left(m_{j}\right)(\mathbf{x}, \mathbf{z}) H_{1}(\mathbf{z}, \mathbf{y})\right) d \mathbf{z}\right| d \mathbf{x} .
$$

If $j \leq 0$, then

$$
\begin{gathered}
M_{j} \leq \int\left|\int H_{1}(\mathbf{x}, \mathbf{z})\left(\pi\left(m_{j}\right)(\mathbf{z}, \mathbf{y})-\pi\left(m_{j}\right)(\mathbf{x}, \mathbf{y})\right) d \mathbf{z}\right| d \mathbf{x} \\
+\int\left|\int\left(\pi\left(m_{j}\right)(\mathbf{x}, \mathbf{y})-\pi\left(m_{j}\right)(\mathbf{x}, \mathbf{z})\right) H_{1}(\mathbf{z}, \mathbf{y}) d \mathbf{z}\right| d \mathbf{x} \\
=J_{1}+J_{2} . \\
J_{1 \leq} \int\left|\int_{d(\mathbf{z}, \mathbf{y}) \leq 8 C_{1} 2^{-j}} H_{1}(\mathbf{x}, \mathbf{z})\left(\pi\left(m_{j}\right)(\mathbf{z}, \mathbf{y})-\pi\left(m_{j}\right)(\mathbf{x}, \mathbf{y})\right) d \mathbf{z}\right| d \mathbf{x} \\
+\int\left|\int_{d(\mathbf{z}, \mathbf{y})>8 C_{1} 2^{-j}} H_{1}(\mathbf{x}, \mathbf{z})\left(\pi\left(m_{j}\right)(\mathbf{z}, \mathbf{y})-\pi\left(m_{j}\right)(\mathbf{x}, \mathbf{y})\right) d \mathbf{z}\right| d \mathbf{x} \\
=J_{11}+J_{12} .
\end{gathered}
$$

By (4.5), (1.2) and (2.13), we have

$$
J_{11} \leq C \int_{d(\mathbf{z}, \mathbf{y}) \leq 8 C_{1} 2^{-j}} \int H_{1}(\mathbf{x}, \mathbf{z}) \frac{2^{j} d(\mathbf{x}, \mathbf{z})}{\left|B\left(\mathbf{y}, 2^{-j}\right)\right|} d \mathbf{x} d \mathbf{z} \leq C 2^{j}
$$

Using (4.4) and (2.13) and (4.5), we obtain 


$$
\begin{aligned}
J_{12} & =\iint_{d(\mathbf{z}, \mathbf{y})>8 C_{1} 2^{-j}} H_{1}(\mathbf{x}, \mathbf{z})\left|\pi\left(m_{j}\right)(\mathbf{x}, \mathbf{y})\right| d \mathbf{z} d \mathbf{x} \\
& \leq \iint_{d(\mathbf{x}, \mathbf{z})>C_{1} 2^{-j}} H_{1}(\mathbf{x}, \mathbf{z})\left|\pi\left(m_{j}\right)(\mathbf{x}, \mathbf{y})\right| d \mathbf{z} d \mathbf{x} \leq C 2^{j} .
\end{aligned}
$$

Similarly,

$$
\begin{aligned}
J_{2} \leq & \int_{d(\mathbf{x}, \mathbf{y}) \leq 8 C_{1} 2^{-j}}\left|\int\left(\pi\left(m_{j}\right)(\mathbf{x}, \mathbf{y})-\pi\left(m_{j}\right)(\mathbf{x}, \mathbf{z})\right) H_{1}(\mathbf{z}, \mathbf{y}) d \mathbf{z}\right| d \mathbf{x} \\
& +\int_{d(\mathbf{x}, \mathbf{y})>8 C_{1} 2^{-j}}\left|\int\left(\pi\left(m_{j}\right)(\mathbf{x}, \mathbf{y})-\pi\left(m_{j}\right)(\mathbf{x}, \mathbf{z})\right) H_{1}(\mathbf{z}, \mathbf{y}) d \mathbf{z}\right| d \mathbf{x}=J_{21}+J_{22}
\end{aligned}
$$

Again, applying (1.2), (4.5), (2.13) and the doubling property of the measure, we get

$$
J_{21} \leq C \int_{d(\mathbf{x}, \mathbf{y})<8 C_{1} 2^{-j}} \int_{\mathbf{X}} \frac{d(\mathbf{y}, \mathbf{z}) 2^{j}}{\left|B\left(\mathbf{x}, 2^{-j}\right)\right|} H_{1}(\mathbf{z}, \mathbf{y}) d \mathbf{z} d \mathbf{x} \leq C 2^{j} .
$$

If $d(\mathbf{x}, \mathbf{y})>8 C_{1} 2^{-j}$ then $\pi\left(m_{j}\right)(\mathbf{x}, \mathbf{y})=0$. Hence, thanks to (2.13) and (4.5), we have

$$
\begin{aligned}
J_{22} & \leq \int_{d(\mathbf{x}, \mathbf{y})>8 C_{1} 2^{-j}} \int_{d(\mathbf{x}, \mathbf{z})<2 C_{1} 2^{-j}}\left|\pi\left(m_{j}\right)(\mathbf{x}, \mathbf{z})\right| H_{1}(\mathbf{z}, \mathbf{y}) d \mathbf{z} d \mathbf{x} \\
& \leq \int_{d(\mathbf{z}, \mathbf{y})>2 C_{1} 2^{-j}} \int_{\mathbf{X}}\left|\pi\left(m_{j}\right)(\mathbf{x}, \mathbf{z})\right| H_{1}(\mathbf{z}, \mathbf{y}) d \mathbf{x} d \mathbf{z} \leq C 2^{j} .
\end{aligned}
$$

If $j>0$, then using (4.3) and Corollary 2.5 we arrive to

$$
\begin{aligned}
M_{j} \leq & \int\left|\int\left(H_{1}(\mathbf{x}, \mathbf{z})-H_{1}(\mathbf{x}, \mathbf{y})\right) \pi\left(m_{j}\right)(\mathbf{z}, \mathbf{y}) d \mathbf{z}\right| d \mathbf{x} \\
& +\int\left|\int \pi\left(m_{j}\right)(\mathbf{x}, \mathbf{z})\left(H_{1}(\mathbf{z}, \mathbf{y})-H_{1}(\mathbf{x}, \mathbf{y})\right) d \mathbf{z}\right| d \mathbf{x} \\
\leq & C \int\left|\int_{d(\mathbf{z}, \mathbf{y})<2 C_{1} 2^{-j}} \frac{2^{-j \gamma}}{|B(\mathbf{x}, 1)|} e^{-c_{0} d(\mathbf{x}, \mathbf{y})^{2}}\right| \pi\left(m_{j}\right)(\mathbf{z}, \mathbf{y}) \mid d \mathbf{z} d \mathbf{x} \\
& +C \int\left|\int_{d(\mathbf{x}, \mathbf{z})<2 C_{1} 2^{-j}}\right| \pi\left(m_{j}\right)(\mathbf{x}, \mathbf{z}) \mid \frac{2^{-j \gamma}}{|B(\mathbf{y}, 1)|} e^{-c_{0} d(\mathbf{x}, \mathbf{y})^{2}} d \mathbf{z} d \mathbf{x} \\
\leq & C 2^{-j \gamma} .
\end{aligned}
$$

Finally, (6.4)-(6.8) imply $\sum_{j=-\infty}^{\infty} M_{j} \leq C<\infty$, which completes the proof of the Lemma.

Acknowledgments This research was initiated when the second author was visiting the Institute of Mathematics of the University of Wrocław in the spring of 2014; the financial support and kind hospitality are gratefully acknowledged. The authors want to thank the referee for her/his helpful comments which improved the presentation of the paper. The first author supported by the Polish National Science Center (Narodowe Centrum Nauki, Grant DEC-2012/05/B/ST1/00672). 
Open Access This article is distributed under the terms of the Creative Commons Attribution 4.0 International License (http://creativecommons.org/licenses/by/4.0/), which permits unrestricted use, distribution, and reproduction in any medium, provided you give appropriate credit to the original author(s) and the source, provide a link to the Creative Commons license, and indicate if changes were made.

\section{Appendix 1: Chang-Fefferman Decomposition}

Our goal in this section is to prove a version of Chang-Fefferman decomposition of compactly supported $B M O(\mathbf{X})$ functions. Then we shall establish some properties of the decomposition. We borrow main ideas from [5].

\section{Dyadic Sets}

Theorem 7.1 (Christ [4]) There exist a collection $\mathcal{B}$ of open sets $\left\{Q_{\alpha}^{k} \subset \mathbb{R}^{n+1}: k \in\right.$ $\left.\mathbb{Z}, \alpha \in I_{k}\right\}$, and constants $\delta \in(0,1), 1 \geq a_{0}>0$, and $C_{3}>0$ such that

$$
\begin{gathered}
\left|\mathbf{X} \backslash \bigcup_{\alpha \in I_{k}} Q_{\alpha}^{k}\right|=0 \quad \forall k . \\
\text { If } \ell \geq k \text { then either } Q_{\beta}^{\ell} \subset Q_{\alpha}^{k} \text { or } Q_{\beta}^{\ell} \cap Q_{\alpha}^{k}=\emptyset .
\end{gathered}
$$

For each $(k, \alpha)$ and each $\ell<k$ there is a unique $\beta$ such that $Q_{\alpha}^{k} \subset Q_{\beta}^{\ell}$.

$$
\operatorname{diam}\left(Q_{\alpha}^{k}\right) \leq C_{3} \delta^{k}
$$

Each $Q_{\alpha}^{k}$ contains some ball $B_{Q_{\alpha}^{k}}=B\left(\mathbf{z}_{\alpha}^{k}, a_{0} \delta^{k}\right)$.

Set $\mathcal{B}_{k}=\left\{Q_{\alpha}^{k}: \alpha \in I_{k}\right\}$ and $\ell(Q)=\delta^{k}$ if $Q \in \mathcal{B}_{k}$. Fix a constant $A_{0}>2$ such that for any $\mathbf{x} \in \mathbf{X}$ and $k \in \mathbb{Z}$ there is $Q \in \mathcal{B}_{k}$ such that $B\left(\mathbf{x}, 8 \delta^{k-1}\right) \subset A_{0} B_{Q}$. Then for any ball $B=B(\mathbf{x}, r)$ denote $A_{0} B=B\left(\mathbf{x}, A_{0} r\right)$ by $\tilde{B}$.

\section{Chang-Fefferman Decomposition}

Lemma 7.2 Suppose that $N_{1} \in \mathbb{N}$ is given. Then every $f \in B M O(\mathbf{X})$ with compact support can be decomposed to

$$
f(\mathbf{x})=\sum_{k \in \mathbb{Z}} \sum_{\alpha \in I_{k}} \lambda_{Q_{\alpha}^{k}} a_{Q_{\alpha}^{k}}(\mathbf{x}),
$$

where

$$
a_{Q_{\alpha}^{k}}(\mathbf{x})=\mathcal{L}^{N_{1}} \widetilde{a}_{Q_{\alpha}^{k}}(\mathbf{x}),
$$




$$
\begin{gathered}
\widetilde{a}_{Q_{\alpha}^{k}} \text { is supported by } B_{Q_{\alpha}^{k}}^{\diamond}=C_{4} B_{Q_{\alpha}^{k}}=B\left(\mathbf{z}_{\alpha}^{k}, C_{4} a_{0} \delta^{k}\right) . \\
\left\|\pi\left(X^{I}\right) \widetilde{a}_{Q_{\alpha}^{k}}\right\|_{\infty} \leq C_{I} \delta^{2 N_{1} k-d(I) k}, \quad\left\|\pi\left(X^{I}\right) a_{Q_{\alpha}^{k}}\right\|_{\infty} \leq C_{I} \delta^{-d(I) k}, \\
\sum_{\widetilde{B_{P}^{\circ}} \widetilde{B_{Q}^{\circ}} \neq \emptyset, \ell(P) \leq \ell(Q)}\left|\lambda_{P}\right|^{2}|P| \leq C|Q|\|f\|_{B M O(\mathbf{X})}^{2} \text { for every } Q \in \mathcal{B},
\end{gathered}
$$

where $C_{I}$ and $C$ are independent of $f$.

Remark 7.3 Let us emphasize that the condition (7.7) replaces the condition

$$
\int a_{Q_{\alpha}^{k}}(\mathbf{x}) W(\mathbf{x}) d \mathbf{x}=0 \text { for all polynomials } W \text { of homogeneous degree }<2 N_{1},
$$

in the Chang-Fefferman decomposition for the classical $B M O$ spaces and for the BMO spaces on homogeneous Lie groups. Actually (7.7) implies (7.11) by the integration by parts, since $\mathcal{L}^{N_{1}} W(\mathbf{x})=0$ for every polynomial $W$ of homogeneous degree $<2 N_{1}$.

Proof of Lemma 7.2 For fixed $N_{1}$ let $\phi, \psi, \eta \in \mathcal{S}(\mathbb{R})$ be real valued functions such that

$$
\begin{gathered}
\phi(\xi)=\psi\left(\xi^{2}\right),|\phi(\xi)| \leq C|\xi|^{2 N_{1}+2} \text { for }|\xi|<1, \phi(\xi)=\xi^{2 N_{1}} \eta(\xi), \\
\operatorname{supp} \widehat{\phi}, \operatorname{supp} \widehat{\eta} \subset(-1,1), \\
\int_{0}^{\infty} \phi(t \xi)^{2} \frac{d t}{t}=1, \quad \forall \xi \neq 0 .
\end{gathered}
$$

Here $\widehat{\phi}$ and $\widehat{\eta}$ denote the Fourier transforms of $\phi$ and $\eta$ respectively. Then there are Schwartz class functions $\phi(\sqrt{L})(\boldsymbol{x})$ and $\eta(\sqrt{L})(\boldsymbol{x})$ on $\mathbb{H}_{n}$ such that $\phi(t \sqrt{L}) f(\boldsymbol{x})=$ $f * \phi(t \sqrt{L})(\boldsymbol{x}), \phi(t \sqrt{L})(\boldsymbol{x})=t^{-D} \phi(\sqrt{L})\left(\delta_{t}^{-1} \boldsymbol{x}\right)$. The same holds for $\eta(t \sqrt{L})(\boldsymbol{x})$. Moreover, it follows from (2.13), (7.13), and the finite propagation of the fundamental solution of the wave equation that the functions $\phi(t \sqrt{L})(\boldsymbol{x})$ and $\eta(t \sqrt{L})(\boldsymbol{x})$ are compactly supported, that is, there is a constant $C^{\prime}>0$ such that

$$
\operatorname{supp} \phi(t \sqrt{L}), \operatorname{supp} \eta(t \sqrt{L}) \subset B\left(0, C^{\prime} t\right) \subset \mathbb{H}_{n},
$$


see $[10,21]$ for details. Consequently, by Lemma 2.1,

$$
\phi(t \sqrt{\mathcal{L}})(\mathbf{x}, \mathbf{y})=\eta(t \sqrt{\mathcal{L}})(\mathbf{x}, \mathbf{y})=0 \text { for } d(\mathbf{x}, \mathbf{y})>C_{5} t \text { with } C_{5}=C^{\prime} C_{1}
$$

Additionally,

$$
\left|\pi\left(X^{I}\right)_{\mathbf{x}} \eta(t \sqrt{\mathcal{L}})(\mathbf{x}, \mathbf{y})\right| \leq C_{I, \eta} t^{-d(I)}|B(\mathbf{y}, t)|^{-1}
$$

with the same estimates on $\pi\left(X^{I}\right)_{\mathbf{x}} \phi(t \sqrt{\mathcal{L}})(\mathbf{x}, \mathbf{y})$.

For $f \in L^{2}(\mathbf{X})$ we have

$$
\begin{aligned}
f(\mathbf{x}) & =\lim _{\varepsilon \rightarrow 0, R \rightarrow \infty} \int_{\varepsilon}^{R} \phi(t \sqrt{\mathcal{L}}) \phi(t \sqrt{\mathcal{L}}) f(\mathbf{x}) \frac{d t}{t} \\
& =\sum_{k \in \mathbb{Z}} \sum_{\alpha \in I_{k}} \int_{\delta^{k}}^{\delta^{k-1}} \int_{Q_{\alpha}^{k}} \phi(t \sqrt{\mathcal{L}})(\mathbf{x}, \mathbf{y})(\phi(t \sqrt{\mathcal{L}}) f)(\mathbf{y}) d \mathbf{y} \frac{d t}{t} .
\end{aligned}
$$

Set

$$
\begin{gathered}
\lambda_{Q_{\alpha}^{k}}=\left(\left|Q_{\alpha}^{k}\right|^{-1} \int_{\delta^{k}}^{\delta^{k-1}} \int_{Q_{\alpha}^{k}}|(\phi(t \sqrt{\mathcal{L}}) f)(\mathbf{y})|^{2} d \mathbf{y} \frac{d t}{t}\right)^{1 / 2}, \\
\widetilde{a}_{Q_{\alpha}^{k}}=\lambda_{Q_{\alpha}^{k}}^{-1} \int_{\delta^{k}}^{\delta^{k-1}} \int_{Q_{\alpha}^{k}} t^{2 N_{1}} \eta(t \sqrt{\mathcal{L}})(\mathbf{x}, \mathbf{y})(\phi(t \sqrt{\mathcal{L}}) f)(\mathbf{y}) d \mathbf{y} \frac{d t}{t}, \\
a_{Q_{\alpha}^{k}}(\mathbf{x})=\mathcal{L}^{N_{1}} \widetilde{a}_{Q_{\alpha}^{k}}(\mathbf{x}) \\
=\lambda_{Q_{\alpha}^{k}}^{-1} \int_{\delta^{k}}^{\delta^{k-1}} \int_{Q_{\alpha}^{k}} \phi(t \sqrt{\mathcal{L}})(\mathbf{x}, \mathbf{y}) \phi(t \sqrt{\mathcal{L}}) f(\mathbf{y}) d \mathbf{y} \frac{d t}{t} .
\end{gathered}
$$

Thus (7.6) and (7.7) hold. Now (7.8) follows from (7.4) and (7.15). Observe that $|B(\mathbf{y}, t)| \sim\left|Q_{\alpha}^{k}\right|$ for $(\mathbf{y}, t) \in Q_{\alpha}^{k} \times\left(\delta^{k}, \delta^{k-1}\right)$. Thus from (7.15) and (7.16) we 
conclude

$$
\begin{aligned}
& \left|\pi\left(X^{I}\right) \widetilde{a}_{Q_{\alpha}^{k}}(\mathbf{x})\right| \\
& \quad \leq C_{I}\left|\lambda_{Q_{\alpha}^{k}}^{-1}\right| \int_{\delta^{k}}^{\delta^{k-1}} \int_{Q_{\alpha}^{k}} t^{2 N_{1}-d(I)}\left|Q_{\alpha}^{k}\right|^{-1}|\phi(t \sqrt{\mathcal{L}}) f(\mathbf{y})| d \mathbf{y} \frac{d t}{t} \\
& \quad \leq C_{I}\left|\lambda_{Q_{\alpha}^{k}}^{-1}\right| \delta^{\left(2 N_{1}-d(I)\right) k}\left(\left|Q_{\alpha}^{k}\right|^{-1} \int_{\delta^{k}}^{\delta^{k-1}} \int_{Q_{\alpha}^{k}}|\phi(t \sqrt{\mathcal{L}}) f(\mathbf{y})|^{2} d \mathbf{y} \frac{d t}{t}\right)^{1 / 2} \\
& \quad \leq C_{I} \delta^{\left(2 N_{1}-d(I)\right) k},
\end{aligned}
$$

which gives (7.9). Finally, for fixed $Q \in \mathcal{B}$,

$$
\underset{\widetilde{B_{P}^{\diamond}} \cap \widetilde{B_{Q}^{\diamond}} \neq \emptyset, \ell(P) \leq \ell(Q)}{ }\left|\lambda_{P}\right|^{2}|P| \leq \int_{\left(A_{0} C_{4}\right) B_{Q}} \int_{0}^{\left(A_{0} C_{4}\right) \ell(Q)}|\phi(t \sqrt{\mathcal{L}}) f(\mathbf{x})|^{2} d \mathbf{x} \frac{d t}{t} .
$$

So to finish the proof of (7.10) it suffices to note that $|\phi(t \sqrt{\mathcal{L}}) f(\mathbf{x})|^{2} d \mathbf{x} \frac{d t}{t}$ is a Carleson measure with the estimate

$$
\int_{B} \int_{0}^{\operatorname{diam} B}|\phi(t \sqrt{\mathcal{L}}) f(\mathbf{x})|^{2} d \mathbf{x} \frac{d t}{t} \leq C|B|\|f\|_{B M O(\mathbf{X})}^{2}
$$

for any ball $B \subset \mathbf{X}$. This fact has a standard proof, which for the reader convenience we present here. First from (7.12) and (2.7) one gets

$$
\int_{\mathbf{X}} \phi(t \sqrt{\mathcal{L}})(\mathbf{x}, \mathbf{y}) d \mathbf{y}=0, \quad \forall \mathbf{x} \in \mathbf{X}
$$

Fix a ball $B$ and decompose

$$
f=(f-c) \chi_{C_{6} B}+(f-c) \chi\left(C_{6} B\right)^{c}+c=f_{1}+f_{2}+c
$$

where $c=\left|C_{6} B\right|^{-1} \int_{C_{6} B} f(\mathbf{x}) d \mathbf{x}, C_{6}=16\left(C_{5}+1\right)$. Applying (7.24) we have $\phi(t \sqrt{\mathcal{L}}) f=\phi(t \sqrt{\mathcal{L}}) f_{1}+\phi(t \sqrt{\mathcal{L}}) f_{2}$. By the John-Nirenberg inequality $\left\|f_{1}\right\|_{L^{2}}^{2} \leq$ $C|B|\|f\|_{B M O(\mathbf{X})}^{2}$ (see e.g., [24, Sect. III]). Consequently,

$$
\int_{B} \int_{0}^{\operatorname{diam} B}\left|\phi(t \sqrt{\mathcal{L}}) f_{1}(\mathbf{x})\right|^{2} d \mathbf{x} \frac{d t}{t} \leq C\left\|f_{1}\right\|_{L^{2}}^{2} \leq C|B|\|f\|_{B M O(\mathbf{X})}^{2} .
$$

Further, thanks to (7.15), $\phi(t \sqrt{\mathcal{L}}) f_{2}(\mathbf{x})=0$ for $\mathbf{x} \in B$ and $0<t \leq \operatorname{diam} B$. Thus (7.23) is proved. 
Let us remark that for $\lambda_{Q}$ defined in (7.18) we have

$$
\left|\lambda_{Q}\right| \leq C\|f\|_{B M O(\mathbf{X})},
$$

since $|\phi(t \sqrt{\mathcal{L}}) f(\mathbf{x})|^{2} d \mathbf{x} \frac{d t}{t}$ is a Carleson measure.

Lemma 7.4 Let $N \in \mathbb{Z}^{+}$be given. Then there exist constants $N_{1} \in \mathbb{Z}^{+}$and $C_{N}>0$ such that for any regular kernel $S$ of order 0 of the form (4.1) and any $a_{Q}$ satisfying (7.7)-(7.9) we have

$$
\begin{aligned}
& \left|\pi(S) a_{Q}(\mathbf{x})\right| \leq C_{N}\left(c_{1}+\left\|m_{0}\right\|_{C^{2 N_{1}}}\right)\left(1+\ell(Q)^{-1} d\left(\mathbf{x}, \mathbf{z}_{Q}\right)\right)^{-2 N}, \\
& \left|\pi(S) a_{Q}(\mathbf{x})-\pi(S) a_{Q}\left(\mathbf{x}^{\prime}\right)\right| \leq C_{N}\left(c_{1}+\left\|m_{0}\right\|_{C^{2 N_{1}+1}}\right) \frac{\ell(Q)^{-1} d\left(\mathbf{x}, \mathbf{x}^{\prime}\right)}{1+\ell(Q)^{-1} d\left(\mathbf{x}, \mathbf{x}^{\prime}\right)} \\
& \times\left(\frac{1+\ell(Q)^{-1} d\left(\mathbf{x}, \mathbf{x}^{\prime}\right)}{1+\ell(Q)^{-1} d\left(\mathbf{x}, \mathbf{z}_{Q}\right)}\right)^{2 N} . \\
& \int_{\mathbf{X}} \pi(S) a_{Q}(\mathbf{x}) d \mathbf{x}=0
\end{aligned}
$$

Proof Thanks to (7.7)-(7.9) without loss of generality we may assume that $c_{1}=0$.

In order to prove (7.27) it suffices, by (7.7)-(7.9), (4.5) and Lemma 4.1, to consider $d\left(\mathbf{x}, \mathbf{z}_{Q}\right)>16 C_{4} a_{0} \ell(Q)$. Then, integration by parts leads to

$$
\begin{aligned}
\left|\pi(S) a_{Q}(\mathbf{x})\right| & =\left|\int\left\{\mathcal{L}_{\mathbf{y}}^{N_{1}} k(\mathbf{x}, \mathbf{y})\right\} \widetilde{a}_{Q}(\mathbf{y}) d \mathbf{y}\right| \\
& \leq\left\|L^{N_{1}} m_{0}\right\|_{L^{\infty}} \int_{B_{Q}^{\diamond}}|B(\mathbf{y}, d(\mathbf{x}, \mathbf{y}))|^{-1} d(\mathbf{x}, \mathbf{y})^{-2 N_{1}}\left|\widetilde{a}_{Q}(\mathbf{y})\right| d \mathbf{y} \\
& \leq C\left\|L^{N_{1}} m_{0}\right\|_{L^{\infty}} \frac{|Q|}{\mid B\left(\mathbf{z}_{Q}, d\left(\mathbf{x}, \mathbf{z}_{Q}\right) \mid\right.} d\left(\mathbf{x}, \mathbf{z}_{Q}\right)^{-2 N_{1}} \ell(Q)^{2 N_{1}} \\
& \leq C\left\|L^{N_{1}} m_{0}\right\|_{L^{\infty}} \frac{\ell(Q)^{2 N_{1}+2}}{d\left(\mathbf{x}, \mathbf{z}_{Q}\right)^{2 N_{1}+2}},
\end{aligned}
$$

where in the last inequality we have used (1.4). The proof of (7.27) is complete.

We now turn to prove (7.28). It suffices to consider $d\left(\mathbf{x}, \mathbf{x}^{\prime}\right) \leq \ell(Q)$, otherwise (7.28) follows from (7.27).

Assume first that $d\left(\mathbf{x}, \mathbf{z}_{Q}\right)>16 C_{4} a_{0} \ell(Q)$. Let $g \in C_{c}^{\infty}(\mathbf{X})$ be such that $g(\mathbf{x})=g\left(\mathbf{x}^{\prime}\right)=1$, supp $g \in B\left(\mathbf{x}, d\left(\mathbf{x}, \mathbf{z}_{Q}\right) / 4\right),\left\|\pi\left(X^{I}\right) g\right\|_{\infty} \leq C_{I} d\left(\mathbf{x}, \mathbf{z}_{Q}\right)^{-d(I)}$. 
Then, integrating by parts and using (1.2) together with (4.8) and (7.9), we obtain

$$
\begin{aligned}
& \left|\pi(S) a_{Q}(\mathbf{x})-\pi(S) a_{Q}\left(\mathbf{x}^{\prime}\right)\right| \\
& \quad=\left|\int_{\mathbf{X}}\left(g(\mathbf{x}) k(\mathbf{x}, \mathbf{y})-g\left(\mathbf{x}^{\prime}\right) k\left(\mathbf{x}^{\prime}, \mathbf{y}\right)\right) \mathcal{L}^{N_{1}} \widetilde{a}_{Q}(\mathbf{y}) d \mathbf{y}\right| \\
& \quad=\left|\int_{\mathbf{X}} \mathcal{L}_{\mathbf{y}}^{N_{1}}\left(g(\mathbf{x}) k(\mathbf{x}, \mathbf{y})-g\left(\mathbf{x}^{\prime}\right) k\left(\mathbf{x}^{\prime}, \mathbf{y}\right)\right) \widetilde{a}_{Q}(\mathbf{y}) d \mathbf{y}\right| \\
& \quad \leq d\left(\mathbf{x}, \mathbf{x}^{\prime}\right) \int_{\mathbf{X}} \sum_{i=1}^{2 n}\left\|\pi\left(X_{i}\right)_{\mathbf{x}} \mathcal{L}_{\mathbf{y}}^{N_{1}}(g(\cdot) k(\cdot, \mathbf{y}))\right\|_{\infty}\left|\widetilde{a}_{Q}(\mathbf{y})\right| d \mathbf{y} \\
& \quad \leq C d\left(\mathbf{x}, \mathbf{x}^{\prime}\right)\left\|m_{0}\right\|_{C^{2 N_{1}+1} d\left(\mathbf{x}, \mathbf{z}_{Q}\right)^{-2 N_{1}-1} \ell(Q)^{2 N_{1}}|Q| \cdot\left|B\left(\mathbf{z}_{Q}, d\left(\mathbf{x}, \mathbf{z}_{Q}\right)\right)\right|^{-1}} \\
& \quad \leq C\left\|m_{0}\right\|_{C^{2 N_{1}+1}} \frac{\ell(Q)^{-1} d\left(\mathbf{x}, \mathbf{x}^{\prime}\right)}{1+\ell(Q)^{-1} d\left(\mathbf{x}, \mathbf{x}^{\prime}\right)}\left(\frac{1+\ell(Q)^{-1} d\left(\mathbf{x}, \mathbf{x}^{\prime}\right)}{1+\ell(Q)^{-1} d\left(\mathbf{x}, \mathbf{z}_{Q}\right)}\right)^{2 N_{1}+n+2}
\end{aligned}
$$

where in the last inequality we have applied (1.4).

Assume now that $d\left(\mathbf{x}, \mathbf{z}_{Q}\right) \leq 16 C_{4} a_{0} \ell(Q)$. According to (1.2) it is enough to prove that

$$
\left|\pi\left(X_{i}\right) \pi(S) a_{Q}(\mathbf{x})\right| \leq C\left\|m_{0}\right\|_{C^{2 N_{1}+1}} \ell(Q)^{-1} \text { for } i=1,2, \ldots, 2 n
$$

Consider $j$ 's such that $2^{-j}>\ell(Q)$. Then, by (4.5) and (7.7)-(7.9), we get

$$
\begin{aligned}
\left|\pi\left(X_{k}\right) \pi\left(m_{j}\right) a_{Q}(\mathbf{x})\right| & =\left|\int \pi\left(X_{k}\right)_{\mathbf{x}} \pi\left(m_{j}\right)(\mathbf{x}, \mathbf{y}) a_{Q}(\mathbf{y}) d \mathbf{y}\right| \\
& \leq C \int_{B\left(\mathbf{x}, C 2^{-j}\right)}\left\|Y_{k} m_{0}\right\|_{\infty} 2^{j}\left|B\left(\mathbf{x}, 2^{-j}\right)\right|^{-1}\left|a_{Q}(\mathbf{y})\right| d \mathbf{y} \\
& \leq C\left\|m_{0}\right\|_{C^{1}} 2^{j}
\end{aligned}
$$

Therefore, $\sum_{2^{-j}>\ell(Q)}\left|\pi\left(X_{k}\right) \pi\left(m_{j}\right) a_{Q}(\mathbf{x})\right| \leq C\left\|m_{0}\right\|_{C^{1}} \ell(Q)^{-1}$.

Consider $2^{-j} \leq \ell(Q)$. By (2.9) and (2.2), we have

$$
\begin{aligned}
\pi\left(X_{k}\right)_{\mathbf{x}} \pi\left(m_{j}\right)(\mathbf{x}, \mathbf{y}) & =-\pi\left(Y_{k} m_{j}\right)=-\pi\left(X_{k} m_{j}+w_{k} X_{2 n+1} m_{j}\right)(\mathbf{x}, \mathbf{y}) \\
& =\pi\left(X_{k}\right)_{\mathbf{y}} \pi\left(m_{j}\right)(\mathbf{x}, \mathbf{y})+\pi\left(X_{2 n+1}\right)_{\mathbf{y}} \pi\left(w_{k} m_{j}\right)(\mathbf{x}, \mathbf{y})
\end{aligned}
$$

Hence, integration by parts and use of (4.3) lead to

$$
\begin{aligned}
\left|\pi\left(X_{k}\right) \pi\left(m_{j}\right) a_{Q}(\mathbf{x})\right| \leq & \left|\int \pi\left(m_{j}\right)(\mathbf{x}, \mathbf{y})\left(\pi\left(X_{k}\right) a_{Q}\right)(\mathbf{y}) d \mathbf{y}\right| \\
& +\left|\int \pi\left(w_{k} m_{j}\right)(\mathbf{x}, \mathbf{y})\left(\pi\left(X_{2 n+1}\right) a_{Q}\right)(\mathbf{y}) d \mathbf{y}\right| \\
\leq & \left|\int \pi\left(m_{j}\right)(\mathbf{x}, \mathbf{y})\left\{\left(\pi\left(X_{k}\right) a_{Q}\right)(\mathbf{y})-\left(\pi\left(X_{k}\right) a_{Q}\right)(\mathbf{x})\right\} d \mathbf{y}\right| \\
& +\left|\int \pi\left(w_{k} m_{j}\right)(\mathbf{x}, \mathbf{y})\left(\pi\left(X_{2 n+1}\right) a_{Q}\right)(\mathbf{y}) d \mathbf{y}\right|
\end{aligned}
$$




$$
\begin{aligned}
\leq & \int_{B\left(\mathbf{x}, C 2^{-j}\right)}\left|\pi\left(m_{j}\right)(\mathbf{x}, \mathbf{y})\right| d(\mathbf{x}, \mathbf{y}) \sum_{i=1}^{2 n}\left\|\pi\left(X_{i} X_{k}\right) a_{Q}\right\|_{\infty} d \mathbf{y} \\
& +\int_{B\left(\mathbf{x}, C 2^{-j}\right)}\left|\pi\left(w_{k} m_{j}\right)(\mathbf{x}, \mathbf{y})\right| \cdot \mid\left(\pi\left(X_{2 n+1}\right) a_{Q}(\mathbf{y}) \mid d \mathbf{y}\right. \\
\leq & C\left\|m_{0}\right\|_{\infty} 2^{-j} \ell(Q)^{-2},
\end{aligned}
$$

since $\left|w_{k} m_{j}\right| \leq C 2^{-j}\left|m_{j}\right|$. So, $\sum_{2^{-j} \leq \ell(Q)}\left|\pi\left(X_{k}\right) \pi\left(m_{j}\right) a_{Q}(\mathbf{x})\right| \leq C\left\|m_{0}\right\|_{\infty} \ell(Q)^{-1}$.

Finally (7.29) is a direct consequence of Lemma 4.2, because $a_{Q}$ is a multiple of a $(1, \infty)$-atom for $H^{1}(\mathbf{X})$.

Corollary 7.5 Assume that for $S_{j}, j=1, \ldots, d$, the condition ( $\star$ ) holds. Suppose that $a_{Q}$ satisfies the conclusions (7.7)-(7.9) of the Chang-Fefferman decomposition. Then there is a constant $C_{N}$ such that given any unit vector $v \in \mathbb{R}^{d}$ there exists $\vec{b} Q$ such that

$$
\begin{gathered}
\left|\vec{b}_{Q}\right| \leq C_{N}\left(1+\frac{d\left(\mathbf{z}_{Q}, \mathbf{x}\right)}{\ell(Q)}\right)^{-2 N} \\
\left|\vec{b}_{Q}(\mathbf{x})-\vec{b}_{Q}(\mathbf{y})\right| \leq C_{11} \frac{d(\mathbf{x}, \mathbf{y})}{\ell(Q)}\left(1+\frac{d\left(\mathbf{z}_{Q}, \mathbf{x}\right)}{\ell(Q)}\right)^{-2 N} \\
\text { for } d(\mathbf{x}, \mathbf{y}) \leq A_{0} \ell(Q), \\
\left|\vec{b}_{Q}(\mathbf{x})-\vec{b}_{Q}(\mathbf{y})\right| \leq C_{11} \frac{d(\mathbf{x}, \mathbf{y})}{\ell(Q)}\left(1+\frac{d(\mathbf{x}, \mathbf{y})}{\ell(Q)}\right)^{N-1}\left(1+\frac{d\left(\mathbf{z}_{Q}, \mathbf{x}\right)}{\ell(Q)}\right)^{-N} \\
\text { for all } \mathbf{x}, \mathbf{y} \in \mathbf{X}
\end{gathered}
$$

Proof Define $\vec{b}_{Q}=\pi(\vec{T})\left(a_{Q}\right)$. Then (7.34), (7.35), and (7.37) follow directly from Lemma 7.4 and property ( $\star$ ). It suffices to prove (7.36). If $d(\mathbf{x}, \mathbf{y}) \leq \ell(Q)$ or $d(\mathbf{x}, \mathbf{y}) \leq$ $3 d\left(\mathbf{x}, \mathbf{z}_{Q}\right)$, then (7.36) is deduced easily from (7.28). Finally assume that $d(\mathbf{x}, \mathbf{y})>$ $\ell(Q)$ and $3 d\left(\mathbf{x}, \mathbf{z}_{Q}\right)<d(\mathbf{x}, \mathbf{y})$. Then $d\left(\mathbf{x}, \mathbf{z}_{Q}\right)<d\left(\mathbf{z}_{Q}, \mathbf{y}\right)$ and, consequently, (7.34) implies (7.36).

\section{Auxiliary Functions}

Let $N_{0}$ be a large integer. For a compactly supported $f \in B M O(\mathbf{X})$ define

$$
\tau_{k}(\mathbf{x})=\sum_{\ell(Q)=\delta^{k}}\left|\lambda_{Q}\right|\left(1+\delta^{-k} d\left(\mathbf{z}_{Q}, \mathbf{x}\right)\right)^{-N_{0}},
$$


where $\lambda_{Q}$ are scalars from the Chang-Fefferman decomposition (see Lemma 7.2). Fix $0<\kappa<1-\delta$, where $\delta$ is from Theorem 7.1 and set

$$
\sigma_{k}(\mathbf{x})=\sum_{j \leq k}(1-\kappa)^{k-j} \tau_{j}(\mathbf{x}) .
$$

Then,

$$
\sigma_{k}(\mathbf{x})=\tau_{k}(\mathbf{x})+(1-\kappa) \sigma_{k-1}(\mathbf{x})
$$

Easily, if $N_{0}$ is sufficiently large, then

$$
\tau_{k}(\mathbf{x})^{2} \leq C \sum_{Q \in \mathcal{B}_{k}} \lambda_{Q}^{2}\left(1+\delta^{-k} d\left(\mathbf{z}_{Q}, \mathbf{x}\right)\right)^{-N_{0}} .
$$

Indeed,

$$
\begin{aligned}
& \sum_{\ell(Q)=\delta^{k}, \ell(P)=\delta^{k}}\left|\lambda_{Q} \lambda_{P}\right|\left(1+\frac{d\left(\mathbf{z}_{Q}, \mathbf{x}\right)}{\delta^{k}}\right)^{-N_{0}}\left(1+\frac{d\left(\mathbf{z}_{P}, \mathbf{x}\right)}{\delta^{k}}\right)^{-N_{0}} \\
\leq & \frac{1}{2} \sum_{\ell(Q)=\delta^{k}, \ell(P)=\delta^{k}}\left|\lambda_{Q}\right|^{2}\left(1+\frac{d\left(\mathbf{z}_{Q}, \mathbf{x}\right)}{\delta^{k}}\right)^{-N_{0}}\left(1+\frac{d\left(\mathbf{z}_{P}, \mathbf{x}\right)}{\delta^{k}}\right)^{-N_{0}} \\
& +\frac{1}{2} \sum_{\ell(Q)=\delta^{k}, \ell(P)=\delta^{k}}\left|\lambda_{P}\right|^{2}\left(1+\frac{d\left(\mathbf{z}_{Q}, \mathbf{x}\right)}{\delta^{k}}\right)^{-N_{0}}\left(1+\frac{d\left(\mathbf{z}_{P}, \mathbf{x}\right)}{\delta^{k}}\right)^{-N_{0}} \\
= & \sum_{\ell(Q)=\delta^{k}}\left|\lambda_{Q}\right|^{2}\left(1+\frac{d\left(\mathbf{z}_{Q}, \mathbf{x}\right)}{\delta^{k}}\right)^{-N_{0}} \sum_{\ell(P)=\delta^{k}}\left(1+\frac{d\left(\mathbf{z}_{P}, \mathbf{x}\right)}{\delta^{k}}\right)^{-N_{0}} \\
\leq & C \sum_{\ell(Q)=\delta^{k}}\left|\lambda_{Q}\right|^{2}\left(1+\frac{d\left(\mathbf{z}_{Q}, \mathbf{x}\right)}{\delta^{k}}\right)^{-N_{0}} .
\end{aligned}
$$

Because $\sum_{\ell(P)=\delta^{k}}\left(1+\delta^{-k} d\left(\mathbf{z}_{P}, \mathbf{x}\right)\right)^{-N_{0}} \leq C$ independently of $k$, provided $N_{0}>3$.

Lemma 7.6 (Christ and Geller [5]) If $N_{0}$ is sufficiently large, then for every compacty supported BMO(X)-function $f$ one has

$$
\begin{gathered}
\left\|\tau_{k}\right\|_{\infty} \leq C_{12}\|f\|_{B M O(\mathbf{X})}, \\
\left\|\sigma_{k}\right\|_{\infty} \leq C_{12}\|f\|_{B M O(\mathbf{X})}, \\
\int_{\tilde{B}_{Q}} \sum_{j \geq k} \sigma_{j}(\mathbf{x}) \tau_{j}(\mathbf{x}) d \mathbf{x} \leq C_{12}|Q|\|f\|_{B M O(\mathbf{X})}^{2} \text { if } \ell(Q)=\delta^{k} .
\end{gathered}
$$


Proof The proof is same as of [5, Lemma 3.3].

Lemma 7.7 There is a constant $C$ such that if $f=\sum \lambda_{Q} a_{Q}$ is the Chang-Fefferman decomposition of a BMO(X)-function $f$ given by the proof of Lemma 7.2 such that supp $f \subset B\left(\mathbf{x}_{0}, 1\right)$, then

$$
\left\|\sum_{\ell(Q) \geq C_{0}} \lambda_{Q} a_{Q}\right\|_{\infty} \leq C \sum_{\delta^{k} \geq C_{0}} \delta^{-k}\|f\|_{B M O(\mathbf{X})} \text { for } C_{0} \geq 1 .
$$

Proof There is a constant $M_{1}$ such that for $k \leq 0$ the number of $Q \in \mathcal{B}_{k}$ such that $\lambda_{Q} \neq 0$ is bounded by $M_{1}$ with $M_{1}$ independent of $\mathbf{x}_{0}$. For such $Q,|Q| \sim\left|B\left(\mathbf{x}_{0}, \delta^{k}\right)\right|$. So, by (7.18) and (7.16),

$$
\begin{aligned}
\left|\lambda_{Q}\right|^{2} & \leq \frac{1}{|Q|} \int_{\delta^{k}}^{\delta^{k-1}} \int_{Q}\|f\|_{L^{1}}^{2} \sup _{t \in\left(\delta^{k}, \delta^{k-1}\right), \mathbf{y}^{\prime} \in Q, \mathbf{z} \in B\left(\mathbf{x}_{0}, 1\right)}\left|\phi(t \sqrt{\mathcal{L}})\left(\mathbf{y}^{\prime}, \mathbf{z}\right)\right|^{2} d \mathbf{y} \frac{d t}{t} \\
& \leq\|f\|_{L^{1}}^{2}\left|B\left(\mathbf{z}_{Q}, c \delta^{k}\right)\right|^{-2} \leq C^{\prime} \delta^{-2 k}\|f\|_{L^{1}}^{2}\left|B\left(\mathbf{x}_{0}, 1\right)\right|^{-2} \\
& \leq C^{\prime \prime} \delta^{-2 k}\|f\|_{B M O(\mathbf{X})}^{2},
\end{aligned}
$$

which implies the lemma.

\section{Appendix 2: Proof of Theorem 5.3}

Proof of Theorem 5.3 Using dilations we may assume without loss of generality that $f$ is supported by $B\left(\mathbf{z}_{B}, 1\right)$. By the Chang-Fefferman decomposition given in the proof of Lemma 7.2 we have

$$
f=\sum_{Q} \lambda_{Q} a_{Q}=\sum_{\ell(Q) \leq 1} \lambda_{Q} a_{Q}+\sum_{\ell(Q)>1} \lambda_{Q} a_{Q}=f_{0}+g_{0} .
$$

It follows from Lemma 7.7 that

$$
\left\|g_{0}\right\|_{L^{\infty}} \leq C^{\prime \prime}\|f\|_{B M O(\mathbf{X})}
$$

Thus, in farther consideration we shall deal with the function $f_{0}=\sum_{\ell(Q) \leq 1} \lambda_{Q} a_{Q}$ with $\lambda_{Q}, a_{Q}$ satisfying (7.7)-(7.10). Remark that there is a constant $C_{10}$ independent of $\mathbf{z}_{B}$ such that that if $\lambda_{Q} \neq 0$ in the decomposition of $f_{0}$, then

$$
Q \subset B_{Q}^{\diamond}=B\left(\mathbf{z}_{Q}, C_{4} a_{0} \ell(Q)\right) \subset B\left(\mathbf{z}_{B}, C_{10}\right), \operatorname{supp} a_{Q} \subset B_{Q}^{\diamond}
$$

$$
\#\left\{Q \in \mathcal{B}_{l}: \lambda_{Q} \neq 0\right\} \leq C \delta^{-3 l} \text { for } l \geq 0
$$


Following [5] our task is to construct, by induction, for each integer $l \geq-1$ functions $\vec{h}_{l}, \vec{g}_{l}$ and $\vec{E}_{l}$ on $\mathbf{X}$ taking values in $\mathbb{C}^{d}$ such that

$$
\begin{gathered}
\vec{S}^{*} \cdot \vec{h}_{l}=\sum_{\ell(Q)=\delta^{l}} \lambda_{Q} a_{Q}, \\
\left|\vec{g}_{l}(\mathbf{x})\right| \equiv 1, \\
\vec{g}_{l}=\vec{g}_{l-1}+\vec{h}_{l}+\vec{E}_{l}, \text { for } l \geq 0, \\
\left\{\vec{g}_{l}\right\} \text { converges in } L_{\text {loc }}^{1} \text { to } \vec{g}^{\infty} \in L^{\infty}(\mathbf{X}), \\
\sum_{l=0}^{\infty} \vec{E}_{l} \text { converges in } L_{\text {loc }}^{1} \text { to } \vec{E}^{0} \in B M O(\mathbf{X}), \\
\left\|\vec{E}^{0}\right\|_{B M O(\mathbf{X})} \leq C\|f\|_{B M O(\mathbf{X})}^{2} .
\end{gathered}
$$

The proofs of the above will be based on the following (simultaneously established) properties of $\vec{h}_{l}, \vec{g}_{l}$, and $\vec{E}_{l}$ :

$$
\begin{gathered}
\vec{h}_{l}=\sum_{\ell(Q)=\delta^{l}} \lambda_{Q} \vec{b}_{Q}, \text { where } \\
\left|\vec{b}_{Q}(\mathbf{x})\right| \leq C_{11}\left(1+\frac{d\left(\mathbf{z}_{Q}, \mathbf{x}\right)}{\ell(Q)}\right)^{-2 N} \\
\left|\vec{b}_{Q}(\mathbf{x})-\vec{b}_{Q}(\mathbf{y})\right| \leq C_{11} \frac{d(\mathbf{x}, \mathbf{y})}{\ell(Q)}\left(1+\frac{d(\mathbf{x}, \mathbf{y})}{\ell(Q)}\right)^{N-1}\left(1+\frac{d\left(\mathbf{z}_{Q}, \mathbf{x}\right)}{\ell(Q)}\right)^{-N} \\
\text { for all } \mathbf{x}, \mathbf{y} \in \mathbf{X}, \\
\left|\vec{b}_{Q}(\mathbf{x})-\vec{b}_{Q}(\mathbf{y})\right| \leq C_{11} \frac{d(\mathbf{x}, \mathbf{y})}{\ell(Q)}\left(1+\frac{d\left(\mathbf{z}_{Q}, \mathbf{x}\right)}{\ell(Q)}\right)^{-2 N} \\
\quad \text { for } d(\mathbf{x}, \mathbf{y}) \leq A_{0} \ell(Q) \\
\left\langle\vec{b}_{Q}(\mathbf{x}), \vec{g}_{l-1}\left(\mathbf{z}_{Q}\right)\right\rangle \equiv 0 \text { for } \ell(Q)=\delta^{l}
\end{gathered}
$$




$$
\left|\vec{g}_{l}(\mathbf{x})-\vec{g}_{l}(\mathbf{y})\right| \leq A_{1} \frac{d(\mathbf{x}, \mathbf{y})}{\delta^{l}}\left(1+\frac{d(\mathbf{x}, \mathbf{y})}{\delta^{l}}\right)^{N-1} \sigma_{l}(\mathbf{x}) \text { for all } \mathbf{x}, \mathbf{y} \in \mathbf{X},
$$

$$
\left|\vec{E}_{l}(\mathbf{x})\right| \leq C_{14} \tau_{l}(\mathbf{x}) \sigma_{l}(\mathbf{x})
$$

$$
\left|\vec{E}_{l}(\mathbf{x})-\vec{E}_{l}(\mathbf{y})\right| \leq C \frac{d(\mathbf{x}, \mathbf{y})}{\delta^{l}}\|f\|_{B M O(\mathbf{X})}^{2} \text { for } d(\mathbf{x}, \mathbf{y}) \leq A_{0} \delta^{l},
$$

where $A_{0}$ is a constant appearing in the definition of $\tilde{B}$ and $A_{1}>C_{11}\left(1-C_{11} C_{12} \varepsilon\right)^{-1}$.

We define $\vec{g}_{-1}(\mathbf{x}) \equiv(1,0, \ldots, 0), \vec{E}_{-1}(\mathbf{x})=\vec{h}_{-1}(\mathbf{x}) \equiv(0, \ldots, 0)$.

Assume that (8.3)-(8.6), (8.9)-(8.16) hold for all $j$ such that $j<l$. From Corollary 7.5 one can deduce that for $Q$ with $\ell(Q)=\delta^{l}$ there exists $\vec{b}_{Q}(\mathbf{x})$ satisfying (8.10)(8.13) such that $a_{Q}(\mathbf{x})=\vec{S}^{*} \cdot \vec{b}_{Q}(\mathbf{x})$. Let $\vec{h}_{l}(\mathbf{x})$ be given by (8.9). Then, thanks to (8.10) and Lemma 7.6,

$$
\left|\vec{h}_{l}(\mathbf{x})\right| \leq C_{11} \tau_{l}(\mathbf{x}) \leq C_{11}\|f\|_{B M O(\mathbf{X})} \leq C_{11} C_{12} \varepsilon
$$

Define $\vec{G}_{l}(\mathbf{x})=\vec{g}_{l-1}(\mathbf{x})+\vec{h}_{l}(\mathbf{x})$. Set $C_{13}=C_{11} C_{12}$. Since $\left|\vec{g}_{l-1}(\mathbf{x})\right| \equiv 1$,

$$
1-C_{13} \varepsilon \leq\left|\vec{G}_{l}(\mathbf{x})\right| \leq 1+C_{13} \varepsilon
$$

In other words $\left|\vec{G}_{l}(\mathbf{x})\right|$ is close to 1 . Thanks to the orthogonality a better estimates is true:

$$
|1-| \vec{G}_{l}(\mathbf{x})|| \leq C_{14} \tau_{l}(\mathbf{x}) \sigma_{l}(\mathbf{x}) \text { with } C_{14}=2(1-\kappa)^{-1} C_{11}^{2} A_{1}
$$

To show (8.19) we estimate

$$
\begin{aligned}
& \left|\sum_{\ell(Q)=\delta^{l}} \lambda_{Q}\left\langle\vec{b}_{Q}(\mathbf{x}), \vec{g}_{l-1}(\mathbf{x})\right\rangle\right| \\
& =\left|\sum_{\ell(Q)=\delta^{l}} \lambda_{Q}\left\langle\vec{b}_{Q}(\mathbf{x}), \vec{g}_{l-1}(\mathbf{x})-\vec{g}_{l-1}\left(\mathbf{z}_{Q}\right)\right\rangle\right| \\
& \leq \sum_{\ell(Q)=\delta^{l}}\left|\lambda_{Q}\right| C_{11}\left(1+\frac{d\left(\mathbf{z}_{Q}, \mathbf{x}\right)}{\ell(Q)}\right)^{-2 N} A_{1} \frac{d\left(\mathbf{x}, \mathbf{z}_{Q}\right)}{\delta^{l-1}}\left(1+\frac{d\left(\mathbf{x}, \mathbf{z}_{Q}\right)}{\delta^{l-1}}\right)^{N-1} \sigma_{l-1}(\mathbf{x}) \\
& \leq C_{11} A_{1} \sum_{\ell(Q)=\delta^{l}}\left|\lambda_{Q}\right|\left(1+\frac{d\left(\mathbf{x}, \mathbf{z}_{Q}\right)}{\delta^{l}}\right)^{-N} \sigma_{l-1}(\mathbf{x}) \\
& \leq C_{11} A_{1} \tau_{l}(\mathbf{x}) \sigma_{l-1}(\mathbf{x}),
\end{aligned}
$$


where in the first inequality we have used (8.10) and (8.14). Recall that $\left|\vec{g}_{l-1}(\mathbf{x})\right| \equiv 1$. Hence,

$$
\begin{aligned}
|1-| \vec{G}_{l}(\mathbf{x})|| & \leq 2\left|\operatorname{Re}\left\langle\vec{h}_{l}(\mathbf{x}), \vec{g}_{l-1}(\mathbf{x})\right\rangle\right|+\left|\vec{h}_{l}(\mathbf{x})\right|^{2} \\
& \leq 2 C_{11} A_{1} \tau_{l}(\mathbf{x}) \sigma_{l-1}(\mathbf{x})+C_{11}^{2} \tau_{l}(\mathbf{x})^{2} \\
& \leq 2 C_{11}^{2} A_{1} \tau_{l}(\mathbf{x})\left(\sigma_{l-1}(\mathbf{x})+\tau_{l}(\mathbf{x})\right) \\
& \leq 2(1-\kappa)^{-1} C_{11}^{2} A_{1} \tau_{l}(\mathbf{x}) \sigma_{l}(\mathbf{x})
\end{aligned}
$$

where in the last inequality we have used (7.40). Thus (8.19) is established.

We define

$$
\begin{aligned}
& \vec{g}_{l}(\mathbf{x})=\frac{\vec{G}_{l}(\mathbf{x})}{\left|\vec{G}_{l}(\mathbf{x})\right|} \\
& \vec{E}_{l}(\mathbf{x})=\vec{g}_{l}(\mathbf{x})-\left(\vec{g}_{l-1}(\mathbf{x})+\vec{h}_{l}(\mathbf{x})\right)=\frac{\vec{G}_{l}(\mathbf{x})}{\left|\vec{G}_{l}(\mathbf{x})\right|}-\vec{G}_{l}(\mathbf{x}) .
\end{aligned}
$$

Our task is to verify (8.14). Using (8.18) we have

$$
\left|\vec{g}_{l}(\mathbf{x})-\vec{g}_{l}(\mathbf{y})\right| \leq\left(1-C_{13} \varepsilon\right)^{-1}\left|\vec{G}_{l}(\mathbf{x})-\vec{G}_{l}(\mathbf{y})\right|
$$

Further,

$$
\left|\vec{G}_{l}(\mathbf{x})-\vec{G}_{l}(\mathbf{y})\right| \leq\left|\vec{g}_{l-1}(\mathbf{x})-\vec{g}_{l-1}(\mathbf{y})\right|+\left|\vec{h}_{l}(\mathbf{x})-\vec{h}_{l}(\mathbf{y})\right| .
$$

By induction the first summand in (8.22) is dominated by

$$
\begin{aligned}
& A_{1} \frac{d(\mathbf{x}, \mathbf{y})}{\delta^{l-1}}\left(1+\frac{d(\mathbf{x}, \mathbf{y})}{\delta^{l-1}}\right)^{N-1} \sigma_{l-1}(\mathbf{x}) \\
& \leq \delta A_{1} \frac{d(\mathbf{x}, \mathbf{y})}{\delta^{l}}\left(1+\frac{d(\mathbf{x}, \mathbf{y})}{\delta^{l}}\right)^{N-1} \sigma_{l-1}(\mathbf{x}) \\
& =\frac{\delta}{1-\kappa} A_{1} \frac{d(\mathbf{x}, \mathbf{y})}{\delta^{l}}\left(1+\frac{d(\mathbf{x}, \mathbf{y})}{\delta^{l}}\right)^{N-1}(1-\kappa) \sigma_{l-1}(\mathbf{x})
\end{aligned}
$$

By (8.11) and (8.9) the second summand in (8.22) is bounded by

$$
\begin{aligned}
& \sum_{\ell(Q)=\delta^{l}}\left|\lambda_{Q}\right|\left|\vec{b}_{Q}(\mathbf{x})-\vec{b}_{Q}(\mathbf{y})\right| \\
& \leq C_{11} \frac{d(\mathbf{x}, \mathbf{y})}{\delta^{l}}\left(1+\frac{d(\mathbf{x}, \mathbf{y})}{\delta^{l}}\right)^{N-1} \sum_{\ell(Q)=\delta^{l}}\left|\lambda_{Q}\right|\left(1+\frac{d\left(\mathbf{z}_{Q}, \mathbf{x}\right)}{\delta^{l}}\right)^{-N} \\
& \leq C_{11} \frac{d(\mathbf{x}, \mathbf{y})}{\delta^{l}}\left(1+\frac{d(\mathbf{x}, \mathbf{y})}{\delta^{l}}\right)^{N-1} \tau_{l}(\mathbf{x}) .
\end{aligned}
$$


Recall that $\delta(1-\kappa)^{-1}<1$. Take $\varepsilon>0$ small enough so that

$$
\frac{\delta}{\left(1-C_{13} \varepsilon\right)(1-\kappa)}<1 .
$$

Recall also that $A_{1}>C_{11}\left(1-C_{13} \varepsilon\right)^{-1}$. By the above

$$
\begin{aligned}
& \left|\vec{g}_{l}(\mathbf{x})-\vec{g}_{l}(\mathbf{y})\right| \\
& \leq\left(1-C_{13 \varepsilon}\right)^{-1}\left|\vec{G}_{l}(\mathbf{x})-\vec{G}_{l}(\mathbf{y})\right| \\
& \leq \frac{d(\mathbf{x}, \mathbf{y})}{\delta^{l}}\left(1+\frac{d(\mathbf{x}, \mathbf{y})}{\delta^{l}}\right)^{N-1} \\
& \quad \times\left(\frac{\delta}{\left(1-C_{13} \varepsilon\right)(1-\kappa)} A_{1}(1-\kappa) \sigma_{l-1}(\mathbf{x})+\frac{C_{11}}{1-C_{13} \varepsilon} \tau_{l}(\mathbf{x})\right) \\
& \leq A_{1} \frac{d(\mathbf{x}, \mathbf{y})}{\delta^{l}}\left(1+\frac{d(\mathbf{x}, \mathbf{y})}{\delta^{l}}\right)^{N-1} \sigma_{l}(\mathbf{x})
\end{aligned}
$$

and (8.14) is established.

To obtain (8.15) note that that thanks to (8.19) we have

$$
\begin{aligned}
\left|\vec{E}_{l}(\mathbf{x})\right| & =\left|\vec{g}_{l}(\mathbf{x})-\vec{G}_{l}(\mathbf{x})\right|=\left|\vec{g}_{l}(\mathbf{x})\left(1-\left|\vec{G}_{l}(\mathbf{x})\right|\right)\right| \\
& =|1-| \vec{G}_{l}(\mathbf{x})|| \leq C_{14} \tau_{l}(\mathbf{x}) \sigma_{l}(\mathbf{x}) .
\end{aligned}
$$

We now turn to prove (8.16). We start by showing that there is a constant $C_{15}$ such that

$$
|| \vec{G}_{l}(\mathbf{x})|-| \vec{G}_{l}(\mathbf{y})|| \leq C_{15} \frac{d(\mathbf{x}, \mathbf{y})}{\delta^{l}} \varepsilon^{2} \text { for } d(\mathbf{x}, \mathbf{y}) \leq A_{0} \delta^{l}
$$

From (8.18) and (8.17) we get

$$
\begin{aligned}
|| \vec{G}_{l}(\mathbf{x})|-| \vec{G}_{l}(\mathbf{y})|| \\
\leq\left.|| \vec{G}_{l}(\mathbf{x})\right|^{2}-\left|\vec{G}_{l}(\mathbf{y})\right|^{2} \mid \\
\leq\left.|| \vec{h}_{l}(\mathbf{x})\right|^{2}-\left|\vec{h}_{l}(\mathbf{y})\right|^{2}|+2|\left\langle\vec{h}_{l}(\mathbf{x}), \vec{g}_{l-1}(\mathbf{x})\right\rangle-\left\langle\vec{h}_{l}(\mathbf{y}), \vec{g}_{l-1}(\mathbf{y})\right\rangle \mid \\
\leq 2 \varepsilon C_{11} C_{12}\left|\vec{h}_{l}(\mathbf{x})-\vec{h}_{l}(\mathbf{y})\right| \\
\quad+2\left|\left\langle\vec{h}_{l}(\mathbf{x}), \vec{g}_{l-1}(\mathbf{x})-\vec{g}_{l-1}(\mathbf{y})\right\rangle\right|+2\left|\left\langle\vec{h}_{l}(\mathbf{x})-\vec{h}_{l}(\mathbf{y}), \vec{g}_{l-1}(\mathbf{y})\right\rangle\right| \\
=J_{1}+J_{2}+J_{3} .
\end{aligned}
$$


Applying (8.9), (8.11) and Lemma 7.6 we obtain

$$
\begin{aligned}
J_{1} & \leq 2 \varepsilon C_{11} C_{12} \sum_{\ell(Q)=\delta^{l}}\left|\lambda_{Q} \| \vec{b}_{Q}(\mathbf{x})-\vec{b}_{Q}(\mathbf{y})\right| \\
& \leq 4 \varepsilon C_{11}^{2} C_{12} \sum_{\ell(Q)=\delta^{l}}\left|\lambda_{Q}\right| \frac{d(\mathbf{x}, \mathbf{y})}{\delta^{l}}\left(1+\frac{d(\mathbf{x}, \mathbf{y})}{\delta^{l}}\right)^{N-1}\left(1+\frac{d\left(\mathbf{z}_{Q}, \mathbf{x}\right)}{\delta^{l}}\right)^{-N} \\
& \leq 4 \varepsilon C_{11}^{2} C_{12}\left(1+A_{0}\right)^{N-1} \frac{d(\mathbf{x}, \mathbf{y})}{\delta^{l}} \tau_{l}(\mathbf{x}) \leq 4 C_{11}^{2} C_{12}^{2}\left(1+A_{0}\right)^{N-1} \frac{d(\mathbf{x}, \mathbf{y})}{\delta^{l}} \varepsilon^{2},
\end{aligned}
$$

since $d(\mathbf{x}, \mathbf{y}) \leq A_{0} \delta^{l}$. To estimate $J_{2}$ we use (8.17), (8.14), and Lemma 7.6 to obtain

$$
\begin{aligned}
J_{2} & \leq 2 \varepsilon C_{11} C_{12} A_{1} \frac{d(\mathbf{x}, \mathbf{y})}{\delta^{l-1}}\left(1+\frac{d(\mathbf{x}, \mathbf{y})}{\delta^{l-1}}\right)^{N-1} \sigma_{l-1}(\mathbf{x}) \\
& \leq 2 C_{11}^{2} C_{12}\left(1+A_{0}\right)^{N-1} A_{1} \frac{d(\mathbf{x}, \mathbf{y})}{\delta^{l}} \varepsilon^{2}
\end{aligned}
$$

In order to estimate $J_{3}$ we apply (8.9) and (8.13) to write

$$
J_{3}=2\left|\left\langle\sum_{\ell(Q)=\delta^{l}} \lambda_{Q}\left(\vec{b}_{Q}(\mathbf{x})-\vec{b}_{Q}(\mathbf{y})\right), \vec{g}_{l-1}(\mathbf{y})-\vec{g}_{l-1}\left(\mathbf{z}_{Q}\right)\right\rangle\right| .
$$

Then utilizing (8.12) and (8.14) we have

$$
\begin{aligned}
J_{3} \leq & 2\left(1+A_{0}\right)^{2 N+1} \\
& \times \sum_{\ell(Q)=\delta^{l}}\left|\lambda_{Q}\right| C_{11} \frac{d(\mathbf{x}, \mathbf{y})}{\delta^{l}}\left(1+\frac{d\left(\mathbf{z}_{Q}, \mathbf{y}\right)}{\delta^{l}}\right)^{-2 N} A_{1} \frac{d\left(\mathbf{y}, \mathbf{z}_{Q}\right)}{\delta^{l}}\left(1+\frac{d\left(\mathbf{y}, \mathbf{z}_{Q}\right)}{\delta^{l}}\right)^{N-1} \sigma_{l}(\mathbf{y}) \\
\leq & 2\left(1+A_{0}\right)^{2 N+1} C_{11} A_{1} \frac{d(\mathbf{x}, \mathbf{y})}{\delta^{l}} \tau_{l}(\mathbf{y}) \sigma_{l}(\mathbf{y}) \\
\leq & 2\left(1+A_{0}\right)^{2 N+1} C_{11} C_{12}^{2} A_{1} \frac{d(\mathbf{x}, \mathbf{y})}{\delta^{l}} \varepsilon^{2},
\end{aligned}
$$

where in the last inequality we have used Lemma 7.6. So the proof of (8.25) is complete.

We are now in a position to finish the proof of (8.16). By the definition of $\vec{E}_{l}$ and (8.18),

$$
\begin{aligned}
& \left|\vec{E}_{l}(\mathbf{x})-\vec{E}_{l}(\mathbf{y})\right| \\
& \quad=\left|\left(\frac{\vec{G}_{l}(\mathbf{x})}{\left|\vec{G}_{l}(\mathbf{x})\right|}-\vec{G}_{l}(\mathbf{x})\right)-\left(\frac{\vec{G}_{l}(\mathbf{y})}{\left|\vec{G}_{l}(\mathbf{y})\right|}-\vec{G}_{l}(\mathbf{y})\right)\right| \\
& \quad=\left|\left(\vec{G}_{l}(\mathbf{y})-\vec{G}_{l}(\mathbf{x})\right)\left(1-\frac{1}{\left|\vec{G}_{l}(\mathbf{x})\right|}\right)+\vec{G}_{l}(\mathbf{y})\left(\frac{1}{\left|\vec{G}_{l}(\mathbf{x})\right|}-\frac{1}{\left|\vec{G}_{l}(\mathbf{y})\right|}\right)\right|
\end{aligned}
$$




$$
\begin{aligned}
\leq & \left(1-C_{13} \varepsilon\right)^{-1}\left|\vec{G}_{l}(\mathbf{x})-\vec{G}_{l}(\mathbf{y})\right||1-| \vec{G}_{l}(\mathbf{x})|| \\
& +\left(1-C_{13} \varepsilon\right)^{-1}|| \vec{G}_{l}(\mathbf{x})|-| \vec{G}_{l}(\mathbf{y})|| \\
\leq & A_{1}\left(1+A_{0}\right)^{N-1} \frac{d(\mathbf{x}, \mathbf{y})}{\delta^{l}} \sigma_{l}(\mathbf{x}) C_{13} \varepsilon+C_{15}\left(1-C_{13} \varepsilon\right)^{-1} \frac{d(\mathbf{x}, \mathbf{y})}{\delta^{l}} \varepsilon^{2},
\end{aligned}
$$

where in the last inequality for the first summand we have used (8.23) while for the second one we have applied (8.25). Now from Lemma 7.6 we obtain (8.16).

Thus the construction of the functions $\vec{h}_{l}, \vec{g}_{l}$ and $\vec{E}_{l}$ satisfying (8.3)-(8.5) and (8.9)-(8.16) is complete.

It remains to prove (8.6)-(8.8). First, (7.29) combined with (8.11) and (8.13) imply

$$
\begin{aligned}
\left|\int_{\mathbf{X}}\left\langle\vec{b}_{Q}(\mathbf{x}), \vec{b}_{P}(\mathbf{x})\right\rangle d \mathbf{x}\right| & \leq C_{17} \frac{\ell(P)}{\ell(Q)}|P|\left(1+\frac{d\left(\mathbf{z}_{Q}, \mathbf{z}_{P}\right)}{\ell(Q)}\right)^{-N} \\
& \text { for } \ell(P) \leq \ell(Q) .
\end{aligned}
$$

Now for nonnegative integers $s_{1} \leq s_{2}$ let $\mathcal{Q}=\mathcal{Q}_{s_{1}, s_{2}}=\bigcup_{l=s_{1}}^{s_{2}} \mathcal{B}_{l}$. In virtue of (8.26),

$$
\begin{aligned}
& \left\|\sum_{l=s_{1}}^{s_{2}} \vec{h}_{l}\right\|_{L^{2}}^{2}=\sum_{Q \in \mathcal{Q}_{s_{1}, s_{2}}} \sum_{P \in \mathcal{Q}_{s_{1}, s_{2}}} \lambda_{Q} \bar{\lambda}_{P} \int_{\mathbf{X}}\left\langle\vec{b}_{Q}(\mathbf{x}), \vec{b}_{P}(\mathbf{x})\right\rangle d \mathbf{x} \\
& \leq 2 C_{17} \sum_{Q \in \mathcal{Q}_{s_{1}, s_{2}}} \sum_{\substack{P \in \mathcal{Q}_{s_{1}, 2} \\
\ell(P) \leq \ell(Q)}}\left|\lambda_{Q}\right|\left|\lambda_{P}\right| \frac{\ell(P)}{\ell(Q)}|P|\left(1+\frac{d\left(\mathbf{z}_{Q}, \mathbf{z}_{P}\right)}{\ell(Q)}\right)^{-N} \\
& \leq 2 C_{17} \sum_{k=0}^{\infty} \sum_{Q \in \mathcal{Q}} \sum_{\substack{P \in \mathcal{Q}_{s_{1}, 2} \\
\ell(P)=\delta^{k} \ell(Q)}}\left|\lambda_{Q}\right|\left|\lambda_{P}\right| \frac{\ell(P)}{\ell(Q)}|P|\left(1+\frac{d\left(\mathbf{z}_{Q}, \mathbf{z}_{P}\right)}{\ell(Q)}\right)^{-N} \\
& \leq 2 C_{18} \sum_{k=0}^{\infty} \sum_{j=0}^{\infty} \sum_{Q \in \mathcal{Q}_{s_{1}, s_{2}}} \sum_{\begin{array}{c}
P \in \mathcal{Q}_{s_{1}, s_{2}} \\
\ell(P)=\delta^{k} \ell(Q) \\
d\left(\mathbf{z}_{Q}, \mathbf{z}_{P}\right) \leq 2^{j} \ell(Q)
\end{array}}\left|\lambda_{Q}\left\|\lambda_{P}\right\| P\right| \delta^{k} 2^{-j N} .
\end{aligned}
$$

Applying two times the Cauchy-Schwarz inequality we obtain

$$
\left\|\sum_{l=s_{1}}^{s_{2}} \vec{h}_{l}\right\|_{L^{2}}^{2} \leq 2 C_{18} \sum_{k=0}^{\infty} \sum_{j=0}^{\infty} \delta^{k} 2^{-j N}\left\{\sum_{Q \in \mathcal{Q}}\left|\lambda_{Q}\right|^{2}|Q|\right\}^{1 / 2}
$$




$$
\begin{aligned}
& \left.\times\left\{\sum_{Q \in \mathcal{Q}} \sum_{\substack{P \in \mathcal{Q} \\
\ell(P)=\delta^{k} \ell(Q) \\
d\left(\mathbf{z}_{Q}, \mathbf{z}_{P}\right) \leq 2^{j} \ell(Q)}}\left|\lambda_{P}\right||P|\right)^{2} \frac{1}{|Q|}\right\}^{1 / 2} \\
& \leq 2 C_{18} \sum_{\substack{k \geq 0 \\
j \geq 0}} \delta^{k} 2^{-j N}\left\{\sum_{Q \in \mathcal{Q}}\left|\lambda_{Q}\right|^{2}|Q|\right\}^{1 / 2} \\
& \left.\times\left\{\sum_{Q \in \mathcal{Q}} \sum_{\begin{array}{c}
P \in \mathcal{Q} \\
\ell(P)=\delta^{k} \ell(Q) \\
d\left(\mathbf{z}_{Q}, \mathbf{z}_{P}\right) \leq 2^{j} \ell(Q)
\end{array}}\left|\lambda_{P}\right|^{2}|P|\right)\left(\sum_{\begin{array}{c}
P \in \mathcal{Q} \\
\ell(P)=\delta^{k} \ell(Q) \\
d\left(\mathbf{z}_{Q}, \mathbf{z}_{P}\right) \leq 2^{j} \ell(Q)
\end{array}}|P|\right) \frac{1}{|Q|}\right\}^{1 / 2} .
\end{aligned}
$$

Observe that for fixed integer $k \geq 0$ and fixed $Q \in \mathcal{Q}$,

$$
\sum_{\substack{\ell(P)=\delta^{k} \ell(Q) \\ d\left(\mathbf{z}_{Q}, \mathbf{z}_{P}\right) \leq 2^{j} \ell(Q)}}|P| \leq C_{19} 2^{3 j}|Q|,
$$

so

$$
\begin{aligned}
\left\|\sum_{l=s_{1}}^{s_{2}} \vec{h}_{l}\right\|_{L^{2}}^{2} \leq & 2 C_{20} \sum_{\substack{k \geq 0 \\
j \geq 0}} \delta^{k} 2^{-j(N-3 / 2)}\left\{\sum_{Q \in \mathcal{Q}}\left|\lambda_{Q}\right|^{2}|Q|\right\}^{1 / 2} \\
& \times\left\{\sum_{\substack { Q \in \mathcal{Q} \\
\begin{subarray}{c}{P \in \mathcal{Q} \\
\ell(P)=\delta^{k} \ell(Q) \\
d\left(\mathbf{z}_{Q}, \mathbf{z}_{P}\right) \leq 2^{j} \ell(Q){ Q \in \mathcal { Q } \\
\begin{subarray} { c } { P \in \mathcal { Q } \\
\ell ( P ) = \delta ^ { k } \ell ( Q ) \\
d ( \mathbf { z } _ { Q } , \mathbf { z } _ { P } ) \leq 2 ^ { j } \ell ( Q ) } }\end{subarray}}\left|\lambda_{P}\right|^{2}|P|\right\}
\end{aligned}
$$

It follows from (1.4) and Theorem 7.1 that there is a constant $C>0$ such that for every $j, k \geq 0$ and every $P \in \mathcal{Q}$ the number of $Q \in \mathcal{Q}$ such that $\ell(P)=\delta^{k} \ell(Q)$, $d\left(\mathbf{z}_{Q}, \mathbf{z}_{P}\right) \leq 2^{j} \ell(Q)$ is bounded by $C 2^{3 j}$. Therefore,

$$
\begin{aligned}
\left\|\sum_{l=s_{1}}^{s_{2}} \vec{h}_{l}\right\|_{L^{2}}^{2} & \leq C_{21} \sum_{\substack{k \geq 0 \\
j \geq 0}} \delta^{k} 2^{-j(N-3)}\left\{\sum_{Q \in \mathcal{Q}}\left|\lambda_{Q}\right|^{2}|Q|\right\}^{1 / 2}\left\{\left(\sum_{P \in \mathcal{Q}}\left|\lambda_{P}\right|^{2}|P|\right)\right\}^{1 / 2} \\
& =C_{22} \sum_{Q \in \mathcal{Q}}\left|\lambda_{Q}\right|^{2}|Q| \leq C_{23} \int_{\delta^{s_{2}}}^{\delta^{s_{1}}} \int_{\mathbf{X}}|\phi(t \sqrt{\mathcal{L}}) f(\mathbf{y})|^{2} d \mathbf{y} \frac{d t}{t}
\end{aligned}
$$


where in the last inequality we have used (7.18). From the spectral theorem we easily conclude that $\left\|\sum_{l=s_{1}}^{s_{2}} \vec{h}_{l}\right\|_{L^{2}}^{2} \rightarrow 0$ as $s_{1}, s_{2} \rightarrow \infty$.

Fix $Q \in \mathcal{B}$. Let $k$ be such that $\delta^{k}=\ell(Q)$. From (8.15) and Lemma 7.6 we obtain

$$
\begin{aligned}
\int_{\tilde{B}_{Q}} \sum_{j \geq k}\left|\vec{E}_{j}(\mathbf{x})\right| d \mathbf{x} & \leq C_{14} \int_{\tilde{B}_{Q}} \sum_{j \geq k} \sigma_{j}(\mathbf{x}) \tau_{j}(\mathbf{x}) d \mathbf{x} \\
& \leq C_{14} C_{12}|Q|\|f\|_{B M O(\mathbf{X})}^{2}
\end{aligned}
$$

On the other hand we conclude from (8.16) that for $\mathbf{x}, \mathbf{y} \in \tilde{Q}$ we have the following estimate on the finite sum:

$$
\begin{aligned}
\sum_{\min (k, 0) \leq j<k}\left|\vec{E}_{j}(\mathbf{x})-\vec{E}_{j}(\mathbf{y})\right| \leq & C d(\mathbf{x}, \mathbf{y})\|f\|_{B M O(\mathbf{X})}^{2} \\
& \times \sum_{\min (k, 0) \leq j<k} \delta^{-j} \leq C\|f\|_{B M O(\mathbf{X})}^{2}
\end{aligned}
$$

From (8.28) and (8.29) we obtain that the series $\sum_{j \geq 0} \vec{E}_{j}(\mathbf{x})$ converges in $L_{\text {loc }}^{1}$ to $\vec{E}^{0}(\mathbf{x})$ and

$$
\frac{1}{\left|\tilde{B}_{Q}\right|} \int_{\tilde{B}_{Q}}\left|\vec{E}^{0}(\mathbf{x})-\sum_{\min (k, 0) \leq j<k} \vec{E}_{j}\left(\mathbf{z}_{Q}\right)\right| d \mathbf{x} \leq C\|f\|_{B M O(\mathbf{X})}^{2},
$$

which gives (8.7) and (8.8).

Since $\sum_{j=0}^{l} \vec{h}_{j}+\vec{E}_{j}=\vec{g}_{l}-\vec{g}_{-1}$, we conclude (8.6) from (8.7), (8.27), (8.28), and (8.4).

Having (8.3)-(8.16) already proved we are in a position to complete the proof of Theorem 5.3.

It follows from (8.24) and Lemma 7.6 that

$$
\left|\vec{E}_{l}(\mathbf{x})\right| \leq C_{12} C_{14}\|f\|_{B M O(\mathbf{X})} \sum_{\ell(Q)=\delta^{l}}\left|\lambda_{Q}\right|\left(1+\frac{d\left(\mathbf{x}, \mathbf{z}_{Q}\right)}{\ell(Q)}\right)^{-N_{0}}
$$

Thus if $d\left(\mathbf{x}, \mathbf{z}_{B}\right)>2 C_{10}$, then thanks to (7.26), (8.1), and (8.2) we have

$$
\begin{aligned}
\sum_{l \geq 0}\left|\vec{E}_{l}(\mathbf{x})\right| & \leq C_{21}\|f\|_{B M O(\mathbf{X})}^{2} \sum_{l \geq 0} \delta^{-3 l}\left(1+\frac{d\left(\mathbf{x}, \mathbf{z}_{B}\right)}{\delta^{l}}\right)^{-N_{0}} \\
& \leq C\|f\|_{B M O(\mathbf{X})}^{2} d\left(\mathbf{x}, \mathbf{z}_{B}\right)^{-N}
\end{aligned}
$$


By (8.27), $\sum_{l \geq 0} \vec{h}_{l}(\mathbf{x})=\sum_{l(Q) \leq 1} \lambda_{Q} \vec{b}_{Q}$ converges in $L^{2}(\mathbf{X})$ and

$$
\begin{aligned}
\left\|\sum_{l \geq 0} \vec{h}_{l}\right\|_{L^{2}(\mathbf{X})} & =\left\|\sum_{\ell(Q) \leq 1} \lambda_{Q} \vec{b} Q\right\|_{L^{2}(\mathbf{X})} \leq C\|f\|_{L^{2}(\mathbf{X})} \\
& \leq C\|f\|_{B M O(\mathbf{X})}\left|B\left(\mathbf{z}_{B}, 1\right)\right|^{1 / 2}
\end{aligned}
$$

We decompose

$$
\begin{aligned}
\sum_{l \geq 0} \vec{h}_{l} & =\sum_{l \geq 0}\left(\vec{g}_{l}-\vec{g}_{l-1}\right)-\sum_{l \geq 0} \vec{E}_{l} \\
& =\vec{g}-\vec{g}_{-1}-\left(\sum_{l \geq 0} \vec{E}_{l}\right) \chi_{B\left(\mathbf{z}_{B}, 2 C_{10}\right)^{c}}-\left(\sum_{l \geq 0} \vec{E}_{l}\right) \chi_{B\left(\mathbf{z}_{B}, 2 C_{10}\right)} .
\end{aligned}
$$

From (8.4) and (8.31) we have

$$
\left\|\vec{g}-\vec{g}_{-1}-\left(\sum_{l \geq 0} \vec{E}_{l}\right) \chi_{B\left(\mathbf{z}_{B}, 2 C_{10}\right)^{c}}\right\|_{L^{\infty}(\mathbf{X})} \leq 2+C\|f\|_{B M O(\mathbf{X})}^{2}
$$

Now using (8.31) combined with (8.8) we get

$$
\left\|\left(\sum_{l \geq 0} \vec{E}_{l}\right) \chi_{B\left(\mathbf{z}_{B}, 2 C_{10}\right)}\right\|_{B M O(\mathbf{X})} \leq C\|f\|_{B M O(\mathbf{X})}^{2}
$$

and, consequently,

$$
\left\|\left(\sum_{l \geq 0} \vec{E}_{l}\right) \chi_{B\left(\mathbf{z}_{B}, 2 C_{10}\right)}\right\|_{L^{2}(\mathbf{X})} \leq C\left|B\left(\mathbf{z}_{B}, 1\right)\right|^{1 / 2}\|f\|_{B M O(\mathbf{X})}^{2} .
$$

Finally, from (8.32) and (8.36) we deduce

$$
\begin{aligned}
& \| \vec{g}-\vec{g}_{-1}-\left(\sum_{l \geq 0} \vec{E}_{l}\right) \chi_{B\left(\mathbf{z}_{B}, 2 C_{10}\right)^{c} \|_{L^{2}(\mathbf{X})}} \\
& =\left\|\sum_{l \geq 0}\right\| \vec{h}_{l}+\left(\sum_{l \geq 0} \vec{E}_{l}\right) \chi_{B\left(\mathbf{z}_{B}, 2 C_{10}\right)} \|_{L^{2}(\mathbf{X})} \\
& \leq C\left|B\left(\mathbf{z}_{B}, 1\right)\right|^{1 / 2}\left(\|f\|_{B M O(\mathbf{X})}+\|f\|_{B M O(\mathbf{X})}^{2}\right) .
\end{aligned}
$$


Let

$$
F=\pi\left(\vec{S}^{*}\right) \cdot\left\{\left(\sum_{l \geq 0} \vec{E}_{l}\right) \chi_{B\left(\mathbf{z}_{B}, 2 C_{10}\right)}\right\} .
$$

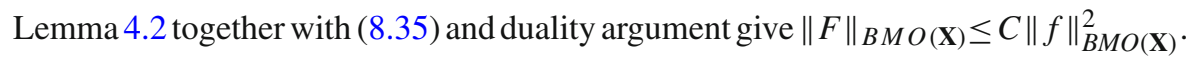
Moreover, $|F(\mathbf{x})| \leq C\|f\|_{B M O(\mathbf{X})}^{2}$ for $d\left(\mathbf{x}, \mathbf{z}_{B}\right)>4 C_{10}$. Putting

$$
\begin{aligned}
& \overrightarrow{\vec{g}}=\vec{g}-\vec{g}_{1}-\left(\sum_{l \geq 0} \vec{E}_{l}\right) \chi_{B\left(\mathbf{z}_{B}, 2 C_{10}\right)^{c}}, \\
& \widetilde{g}_{0}=g_{0}-F \chi_{B\left(\mathbf{z}_{B}, 4 C_{10}\right)^{c}}, \\
& f_{1}=-F \chi_{B\left(\mathbf{z}_{B}, 4 C_{10}\right)}
\end{aligned}
$$

we obtain the required decomposition.

\section{References}

1. Anker, J-Ph, Ben Salem, N., Dziubański, J., Hamba, N.: The Hardy space $H^{1}$ in the rational Dunkl setting. Constr. Approx. 42, 93-128 (2015)

2. Burkholder, D.L., Gundy, R.F., Silverstein, M.L.: A maximal function characterization of the class $H^{p}$. Trans. Am. Math. Soc. 157, 137-153 (1971)

3. Christ, M.: On regularity of inverses of singular integral operators Duke. Math. J. 57, 547-598 (1984)

4. Christ, M.: A $T(b)$ theorem with remarks on analytic capacity and the Cauchy integral. Colloq. Math. 60(61), 601-628 (1990)

5. Christ, M., Geller, D.: Singular integral characterizations of Hardy spaces on homogeneous groups. Duke Math. J. 51, 547-598 (1984)

6. Coifman, R.: A real variable characterization of $H^{p}$. Studia Math. 51, 269-274 (1974)

7. Coifman, R., Weiss, G.: Analyse Harmonique Non-commutative sur Certains Espaces Homogènes. Lecture Notes in Mathematics. Étude de certaines intégrales singulières, vol. 242. Springer, Berlin (1971)

8. Coifman, R., Weiss, G.: Extensions of Hardy spaces and their use in analysis. Bull. Am. Math. Soc. 83, 569-615 (1977)

9. Coifman, R., Weiss, G.: Transference Methods in Analysis. CBMS Regional Conference Series in Mathematics, vol. 31. AMS, Providence (1977)

10. Coulhon, T., Sikora, A.: Gaussian heat kernel upper bounds via Phragmen-Lindelof theorem. Proc. Lond. Math. Soc. (3) 96, 507-544 (2008)

11. Fefferman, C., Stein, E.M.: $H^{p}$ spaces of several variables. Acta Math. 129, 137-193 (1972)

12. Folland, D.G., Stein, E.M.: Hardy Spaces on Homogeneous Groups. Princeton University Press, Princeton (1982)

13. Hofmann, S., Lu, G.Z., Mitrea, D., Mitrea, M., Yan, L.X.: Hardy Spaces Associated with Non-negative Self-adjoint Operators Satisfying Davies-Gafney Estimates. Memoirs of the American Mathematical Society 214, vol. 1007. American Mathematical Society, Providence (2011)

14. Hulanicki, A.: A functional calculus for Rockland operators on nilpotent Lie groups. Studia Math. 78, 253-266 (1984)

15. Jotsaroop, K.: Grushin Multiplier and Toeplitz Operators. PhD thesis, Department of Mathematics Indian Institute of Sciences, Bangalore (2012)

16. Jotsaroop, K., Sanjay, P.K., Thangavelu, S.: Riesz transforms and multipliers for the Grushin operator. J. Anal. Math. 119, 255-273 (2013) 
17. Macías, R.A., Segovia, C.: A decomposition into atoms of distributions on spaces of homogeneous type. Adv. Math. 33, 271-309 (1979)

18. Martini, A., Sikora, A.: Weighted Plancherel estimates and sharp spectral multipliers for the Grushin operators. Math. Res. Lett. 19, 1075-1088 (2012)

19. Meyer, R.: $L^{p}$ estimates for the wave equation associated to the Grushin operator. $\mathrm{PhD}$ thesis, Mathematisch-Naturwissenschaftlichen Fakultät, Christian-Albrechts-Universität, Kiel, Germany (2006)

20. Robinson, D.W., Sikora, A.: Analysis of degenerate elliptic operators of Grushin type. Math. Z. 260, 475-508 (2008)

21. Sikora, A.: Riesz transform, Gaussian bounds and the method of wave equation. Math. Z. 247, 643-662 (2004)

22. Stein, E.M.: Harmonic Analysis, Real-Variable Methods, Orthogonality, and Oscillatory Integrals. Princeton University Press, Princeton (1993)

23. Stein, E.M., Weiss, G.: On the theory of harmonic functions of several variables, I: the theory of $H^{p}$ spaces. Acta Math. 103, 25-62 (1960)

24. Stromberg, J.-O., Torchinsky, A.: Weighted Hardy Spaces. Lecture Notes in Mathematics, vol. 1381. Springer, Berlin (1989)

25. Uchiyama, A.: A maximal function characterization of $H^{p}$ on the space of homogeneous type. Trans. Am. Math. Soc. 262(2), 579-592 (1980)

26. Uchiyama, A.: A constructive proof of the Fefferman-Stein decomposition of BMO $\left(\mathbb{R}^{n}\right)$. Acta Math. 148, 215-241 (1982)

27. Varopoulos, NTh, Saloff-Coste, L., Coulhon, T.: Analysis and Geometry on Groups. Cambridge Univesrity Press, Cambridge (1992) 